TRANSACTIONS OF THE

AMERICAN MATHEMATICAL SOCIETY

Volume 361, Number 8, August 2009, Pages 4405-4436

S 0002-9947(09)04671-6

Article electronically published on March 13, 2009

\title{
IGUSA-TYPE FUNCTIONS ASSOCIATED TO FINITE FORMED SPACES AND THEIR FUNCTIONAL EQUATIONS
}

\author{
BENJAMIN KLOPSCH AND CHRISTOPHER VOLL
}

\begin{abstract}
We study symmetries enjoyed by the polynomials enumerating non-degenerate flags in finite vector spaces, equipped with a non-degenerate alternating bilinear, Hermitian or quadratic form. To this end we introduce Igusa-type rational functions encoding these polynomials and prove that they satisfy certain functional equations.

Some of our results are achieved by expressing the polynomials in question in terms of what we call parabolic length functions on Coxeter groups of type $A$. While our treatment of the orthogonal case exploits combinatorial properties of integer compositions and their refinements, we formulate a precise conjecture how in this situation, too, the polynomials may be described in terms of parabolic length functions.
\end{abstract}

\section{InTRODUCTION AND DISCUSSION OF RESULTS}

1.1. General introduction. Counting functions occurring naturally in algebra and geometry frequently display symmetries which manifest themselves in the form of certain functional equations. A classical example is the functional equation satisfied by the Weil zeta function of a smooth, projective algebraic variety defined over a finite field. More recently, Stanley established similar symmetries for the Hilbert-Poincaré series of graded algebras, with remarkable applications to counting problems in combinatorics and topology; see [17. The symmetries of the Weil zeta functions lie at the heart of Denef and Meuser's proof of a functional equation for certain $p$-adic integrals, called Igusa's local zeta functions; see [3]. The phenomenon of functional equations also arises in the context of zeta functions associated to groups and rings. Indeed, a recent result of the second author establishes functional equations for local zeta functions of finitely generated nilpotent groups, enumerating the numbers of prime-power index subgroups; see 20. It depends on generalisations

Received by the editors August 7, 2006 and, in revised form, October 25, 2007.

2000 Mathematics Subject Classification. Primary 05E15; Secondary 15A63, 20 F55.

Key words and phrases. Finite formed spaces, Coxeter groups, zeta functions, functional equations.

The results in this paper form part of the first author's Habilitation thesis at the University of Düsseldorf. The second author acknowledges support by the Deutsche Forschungsgemeinschaft and the Max-Planck-Gesellschaft. He gratefully acknowledges the hospitality of the HeinrichHeine-Universität in Düsseldorf and the Max-Planck-Institut für Mathematik in Bonn during the writing of this paper. This paper forms part of his Habilitation thesis at the University of Düsseldorf.

(C)2009 Benjamin Klopsch and Christopher Voll 
of methods developed for studying Igusa's local zeta functions and draws on some of the ideas introduced in the current paper 1

In the current paper we are concerned with a counting problem in geometric algebra: we study the numbers of flags of non-degenerate subspaces in a finite vector space, equipped with an alternating bilinear, Hermitian or quadratic form. The polynomials giving these numbers can easily be computed, e.g. by following Artin's classical book [1] on Geometric Algebra. Combining ideas from the theory of zeta functions, Coxeter groups and combinatorics, we are able to establish remarkable symmetries satisfied by these numbers which are far from evident from the formulae. This is achieved by proving functional equations for rational functions encoding the polynomials in question; see Theorems $\mathrm{A}$ and $\mathrm{B}$ below. A recurrent idea in the present paper is to describe these polynomials in terms of Coxeter groups and then to deduce functional equations from generalisations of arguments of Igusa. In an important special case of Theorem A we can only conjecture such a description, leading to Conjecture $\mathrm{C}$, which is of independent interest. The proof we give for this special case of Theorem $\mathrm{A}$ is combinatorial.

Our results have a precedent in the work of Igusa. In [11, Part II] Igusa establishes a formalism for studying certain $p$-adic integrals associated to reductive algebraic groups. Igusa computes a closed formula for these integrals in terms of the associated Weyl groups and their root systems. In the case of classical groups the data involved encode the numbers of flags of totally isotropic subspaces in finite polar spaces. In this sense our work is complementary to that of Igusa. In both cases, the functional equations may be understood in terms of the symmetry which is given by (right-)multiplication by the longest element in a Coxeter group.

Igusa's functions are closely related to $p$-adic integrals associated to zeta functions of groups and rings, where many instances of functional equations similar to the ones described in Theorem $\mathrm{A}$ occur. The analytic properties of Euler products of $p$-adic integrals of this type are also objects of intense study. We refer the reader to [4, 5, 17, 18, 6, 20] for more information on analytic properties of zeta functions of groups and their functional equations. Whilst we first encountered some of the Igusa-type rational functions studied in the current paper in the context of zeta functions of groups, they themselves are not generating functions. Rather than encoding infinite arithmetic sequences they are associated to finite formed spaces. We remark that a priori the rational functions studied in the current paper do not have a natural interpretation as $p$-adic integrals.

\subsection{Detailed statement of results.}

1.2.1. Theorem $\mathrm{A}$, In order to give a detailed statement of our results, we require some notation. We fix a natural number $n \in \mathbb{N}$ and consider $n$-dimensional vector spaces $V$ over a finite field $F$, equipped with a non-degenerate

- alternating bilinear form $B$ (the 'symplectic case'),

- Hermitian form $B$ (the 'unitary case'), or

- quadratic form $f$ (the 'orthogonal case').

In the symplectic and unitary cases, we formally define $f: V \rightarrow F$ by $f(x):=$ $B(x, x)$. In the orthogonal case, we let $B$ denote the bilinear form obtained by polarising $f$ : if $\operatorname{char} F \neq 2$, then $B$ is non-degenerate symmetric, whereas, if $\operatorname{char} F=2$,

\footnotetext{
${ }^{1}$ The last two sentences were added during the revision of the manuscript in October 2007. In the current version we inserted references to [20] and [6] where appropriate.
} 
then $B$ is alternating and possibly degenerate. The triple $\mathcal{V}:=(V, B, f)$ will be called a formed space. We also introduce a parameter $\gamma$ equal to 1 in the unitary case and equal to $1 / 2$ otherwise; with this convention, $F=\mathbb{F}_{q^{2 \gamma}}$ for a prime power $q$.

Recall that, by the classification of finite formed spaces (cf., e.g., [2, Section 3.3]), $\mathcal{V}$ decomposes as an orthogonal direct sum of a certain number of hyperbolic planes and an anisotropic space of dimension $d \in\{0,1,2\}$. In the orthogonal case we attach a sign $\varepsilon \in\{-1,1\}$ to $\mathcal{V}$ if $n$ is even, according to whether $d$ equals 0 or 2 . The six possibilities are given by Table 1 .

TABLE 1

\begin{tabular}{|c|c|c|c|c|}
\hline geometric type & $n$ & $d$ & $\varepsilon$ & $\gamma$ \\
\hline symplectic & $2 m$ & 0 & - & $1 / 2$ \\
unitary & $2 m$ & 0 & - & 1 \\
unitary & $2 m+1$ & 1 & - & 1 \\
orthogonal & $2 m$ & 0 & 1 & $1 / 2$ \\
orthogonal & $2 m+1$ & 1 & - & $1 / 2$ \\
orthogonal & $2 m$ & 2 & -1 & $1 / 2$ \\
\hline
\end{tabular}

In the current paper we study rational functions incorporating the numbers of $F$-rational points of the varieties of flags of non-degenerate subspaces in $\mathcal{V}$. We write $[n-1]$ for $\{1, \ldots, n-1\}$. By a non-degenerate flag of type $J=\left\{j_{1}, \ldots, j_{s}\right\} \subseteq$ $[n-1]$, where $j_{1}<j_{2}<\cdots<j_{s}$, we mean a family $\mathbf{U}_{J}=\left(U_{j}\right)_{j \in J}$ of non-degenerate subspaces of $\mathcal{V}$ with $U_{j_{1}} \subset \cdots \subset U_{j_{s}}$ and $\operatorname{dim} U_{j}=j$ for each $j \in J$. Let

$$
a_{\mathcal{V}}^{J}(q):=\mid\left\{\mathbf{U}_{J} \mid \mathbf{U}_{J} \text { a non-degenerate flag of type } J\right\} \mid .
$$

Then $a_{\mathcal{V}}^{J}(q)$ is a monic polynomial in $q$ (cf. the remarks at the end of this subsection regarding the orthogonal case), and we set

$$
\alpha_{\mathcal{V}}^{J}\left(q^{-1}\right):=a_{\mathcal{V}}^{J}(q) / q^{\operatorname{deg}_{q} a_{\mathcal{V}}^{J}}
$$

We encode these numbers in rational functions as follows. Let $\mathbf{X}=\left(X_{i}\right)_{i}$ be a finite family of independent indeterminates. Fix a family of rational functions $\mathbf{F}=\left(F_{J}(\mathbf{X})\right)_{J \subseteq[n-1]}$ in $\mathbf{X}$ with the inversion property that

$$
\text { for all } I \subseteq[n-1]: F_{I}\left(\mathbf{X}^{-1}\right)=(-1)^{|I|} \sum_{J \subseteq I} F_{J}(\mathbf{X}) .
$$

A simple and naturally occurring example (cf. [11, Part II]) of a family with this property is $\left(\prod_{j \in J} \frac{X_{j}}{1-X_{j}}\right)_{J \subseteq[n-1]}$. By defining

$$
\operatorname{Ig}_{\mathcal{V}}\left(q^{-1}, \mathbf{X}\right):=\operatorname{Ig}_{\mathcal{V}, \mathbf{F}}\left(q^{-1}, \mathbf{X}\right):=\sum_{J \subseteq[n-1]} \alpha_{\mathcal{V}}^{J}\left(q^{-1}\right) F_{J}(\mathbf{X})
$$

we associate to $\mathcal{V}$ and $\mathbf{F}$ a rational function in $q$ and the variables $\left(X_{i}\right)_{i}$. Some explicit examples of these Igusa-type functions may be found in the Appendix. 
The first main result of this paper is

Theorem A. For each $n$-dimensional, non-degenerate formed space $\mathcal{V}$ the associated Igusa-type function satisfies the functional equation

$$
\operatorname{Ig}_{\mathcal{V}}\left(q, \mathbf{X}^{-1}\right)=(-1)^{a} q^{b} \operatorname{Ig}_{\mathcal{V}}\left(q^{-1}, \mathbf{X}\right)
$$

where the integers $a$ and $b$ are given by Table 2 (with $m:=\left\lfloor\frac{n}{2}\right\rfloor$ ).

TABLE 2

\begin{tabular}{|c|c|c|c|c|}
\hline geometric type & $n$ & $\varepsilon$ & $a$ & $b$ \\
\hline symplectic & $2 m$ & - & $m-1$ & $m(m-1)$ \\
unitary & $n$ & - & $\left(\begin{array}{l}n \\
2\end{array}\right)+n-1$ & $\left(\begin{array}{l}n \\
2\end{array}\right)$ \\
orthogonal & $2 m$ & 1 & $m+1$ & $m^{2}$ \\
orthogonal & $2 m+1$ & - & $m$ & $m(m+1)$ \\
orthogonal & $2 m$ & -1 & $m$ & $m^{2}$ \\
\hline
\end{tabular}

As we explained in the general introduction, we see Theorem $\mathrm{A}$ primarily as a result pertaining to the polynomials $\alpha_{\mathcal{V}}^{J}\left(q^{-1}\right)$; the choice of the family of rational functions $\mathbf{F}$ is secondary. The inversion property (IP) satisfied by the rational functions $\mathbf{F}$ is a key ingredient which we require for our subsequent combinatorial and group theoretical considerations. We remark that rational functions $\mathbf{F}$ satisfying the inversion property arise naturally in Igusa's work as well as in the context of zeta functions of groups and rings; cf. [19, 20.

Remark 1.1 (Analogy with Igusa's work). The analogy with Igusa's paper [1] is the following. In [11, p. 706] Igusa gives a formula for a $p$-adic integral, essentially of the form

$$
Z(s)=\frac{\sum_{w \in W} q^{-l(w)} \prod_{\alpha_{j} \in w\left(R^{-}\right)} q^{A_{j}-B_{j} s}}{\prod_{j=1}^{\ell}\left(1-q^{A_{j}-B_{j} s}\right)},
$$

where $q$ is a prime power, $W$ is a Weyl group, $l$ denotes the standard Coxeter length function, $S=\left\{\alpha_{1}, \ldots, \alpha_{\ell}\right\}$ constitutes a basis for the root system, $R^{-}$denotes the set of negative roots with respect to $S$, the parameters $A_{j}, B_{j}$ are suitable integers and $s$ is a complex variable. It is immediate that

$$
Z(s)=\sum_{J \subseteq[\ell]} \beta_{J}\left(q^{-1}\right) F_{J}(\mathbf{X}),
$$

where

$$
\beta_{J}\left(q^{-1}\right):=\sum_{\substack{w \in W \\ D(w) \subseteq I}} q^{-l(w)}, \quad D(w):=\left\{j \in[\ell] \mid \alpha_{j} \in w\left(R^{-}\right)\right\}
$$

and

$$
F_{J}(\mathbf{X}):=\prod_{j \in J} \frac{X_{j}}{1-X_{j}}, \quad X_{j}:=q^{A_{j}-B_{j} s} .
$$

The sets $D(w)$ may be interpreted as descent sets (cf. Section 2). If $W$ comes from a classical group, the polynomials $\beta_{J}\left(q^{-1}\right)$ which arise in this way carry a geometric meaning: the number $b_{J}(q)$ of flags of totally isotropic subspaces in an associated finite polar space equals $\beta_{J}(q)$. In this case $b_{J}(q)$ gives the number of $\mathbb{F}_{q}$-points of a smooth projective variety, and we have $b_{J}(q) / q^{\operatorname{deg}_{q}\left(b_{J}\right)}=b_{J}\left(q^{-1}\right)=\beta_{J}\left(q^{-1}\right)$. In 
this sense the rational functions $\operatorname{Ig}_{\mathcal{V}}$ studied in the current paper, which are built in a similar way from the numbers of non-degenerate flags, complement Igusa's function $Z(s)$.

The key to Igusa's functional equation is to interpret the inversion of the 'variable' $q$ in terms of a natural symmetry of the root system of the Weyl group $W$. This symmetry arises from (right-)multiplication by the longest element $w_{0} \in W$. A recurrent theme of the present paper is to give a suitable description of the polynomials $\alpha_{\mathcal{V}}^{J}\left(q^{-1}\right)$ in terms of Coxeter groups. Based on such a description one can then follow Igusa's approach to derive functional equations. We note that, contrary to the situation studied by Igusa, the 'normalisation' of the polynomials $a_{\mathcal{V}}^{J}(q)$ is indispensable (unlike their counterparts $\beta_{J}$ and $b_{J}$, the polynomials $\alpha_{\mathcal{V}}^{J}$ and $a_{\mathcal{V}}^{J}$ are typically not equal); cf. the examples given in the Appendix. Moreover, it is worth noting that as a side effect of passing to the normalised polynomials $\alpha_{\mathcal{V}}^{J}\left(q^{-1}\right)$ the assumption that $\mathcal{V}$ is non-degenerate means no loss of generality.

We now discuss the proof of Theorem $\mathrm{A}$. In the symplectic and unitary case it follows from Witt's Extension Theorem that the respective isometry group acts transitively on the non-degenerate flags of a given type. A simple stabiliser computation reveals that the polynomials $\alpha_{\mathcal{V}}^{J}\left(q^{-1}\right)$ may be expressed in terms of Gaussian polynomials (or $q$-binomial coefficients), which in turn admit a well-known description in terms of the length function on a Coxeter group of type $A_{\gamma n-1}$. The functional equation then follows with the same argument which Igusa has given in [11, Part II]. It rests on the fact that, in a Coxeter group, the effect of rightmultiplication by the longest element on an element's length and descent set is well understood.

In the orthogonal case, however, things are more intricate. To begin with, the $a_{\mathcal{V}}^{J}(q)$ flags of type $J$ come in up to $2^{|J|}$ isomorphism types and counting them together seems to be crucial for the occurrence of a functional equation. But of course the natural action of the respective orthogonal group on these flags is not transitive. The proof we give for this case of Theorem $\mathrm{A}$ is based on a combinatorial analysis of the polynomials $\alpha_{\mathcal{V}}^{J}\left(q^{-1}\right)$ in terms of integer compositions and their refinements. Complementing this approach, we propose in Conjecture $\mathrm{C}$ an explicit formula which expresses these polynomials, too, in terms of Coxeter group data.

Remark 1.2 (The orthogonal case in characteristic 2). As is well known, quadratic forms are intimately related to symmetric bilinear forms. In fact, over a field of characteristic not equal to 2 , the two notions lead to one and the same theory: a quadratic space $\mathcal{V}=(V, B, f)$ over a field $F$ with char $F \neq 2$ can equally well be regarded as a symmetric bilinear space and vice versa. Such a space $\mathcal{V}$ is said to be non-degenerate if the bilinear form $B$ is non-degenerate, i.e. if the radical $\operatorname{Rad}(B):=\{x \in V \mid \forall y \in V: B(x, y)=0\}$ is the zero subspace. In particular, enumerating non-degenerate flags in a quadratic space $\mathcal{V}$ is the same as counting non-degenerate flags in the symmetric bilinear space $\mathcal{V}$.

In characteristic 2 , however, one has to distinguish more carefully between quadratic and symmetric bilinear forms. It is noteworthy that the analogous statement of Theorem A for symmetric bilinear spaces does not hold in characteristic 2: in the Appendix we display a 4-dimensional non-degenerate symmetric bilinear space whose associated 'Igusa-type' function does not satisfy a functional equation.

Now consider quadratic spaces $\mathcal{V}=(V, B, f)$ over a field $F$ with char $F=2$. In this context $B$ is alternating and carries less information than $f$. There are basically 
two notions of 'non-degeneracy', but unfortunately no standard terminology; cf. 2], [9], 13, Appendix 1], 14]. In this paper, we call $\mathcal{V}$ non-defective if the associated bilinear form $B$ is non-degenerate, i.e. if the radical $\operatorname{Rad}(B):=\{x \in V \mid \forall y \in V$ : $B(x, y)=0\}$ is the zero subspace. This can be thought of as a strong version of 'nondegeneracy'; in particular, every non-defective quadratic space is even-dimensional. But enumerating non-defective flags in a quadratic space over $F$ is the same as counting non-degenerate flags in the induced alternating bilinear space, so we gain nothing new. We call a quadratic space $\mathcal{V}$ non-degenerate if the restriction of $f$ to the $\operatorname{radical} \operatorname{Rad}(B)$ is anisotropic, i.e. if for all $x \in \operatorname{Rad}(B)$ either $x=0$ or $f(x) \neq 0$. This concept of 'non-degeneracy' is more flexible; in particular, there are non-degenerate quadratic spaces of any given dimension. Moreover, this turns out to be the right notion to formulate Theorem $\mathrm{A}$. In fact, the polynomials $a_{\mathcal{V}}^{J}(q)$ counting non-degenerate flags of type $J$ are the same in all characteristics; see Section 4 .

1.2.2. Theorem $\mathrm{B}$, In the symplectic and unitary case, we prove a result which is slightly more general than Theorem A Rather than counting flags which are nondegenerate with respect to a single non-degenerate sesquilinear form $B$, we study the numbers of flags which are non-degenerate with respect to a 'flag of forms'. Loosely speaking, a flag of sesquilinear forms $\boldsymbol{B}$ of type $I \subseteq[n-1]$ is a family of sesquilinear forms such that

- all but the first form are degenerate,

- each but the last form is defined on the radical of its successor,

- the last form is defined on the total space $V$ and

- the non-zero radicals constitute a flag of type $I$ in $V$.

'Non-degeneracy' is defined inductively; see Section 3 for details.

Now let $\boldsymbol{B}$ be a sesquilinear flag of forms of type $I \subseteq[n-1]$ on $V$. We denote by $a_{(V, \boldsymbol{B})}^{J}(q)$ the number of flags of type $J \subseteq[n-1]$ which are non-degenerate with respect to $\boldsymbol{B}$. In the symplectic case, both the type $I$ of $\boldsymbol{B}$ and all the sets $J \subseteq[n-1]$ for which $a_{(V, \boldsymbol{B})}^{J}(q)$ is non-zero necessarily consist of even numbers. From the normalised polynomials

$$
\alpha_{(V, \boldsymbol{B})}^{J}\left(q^{-1}\right):=a_{(V, \boldsymbol{B})}^{J}(q) / q^{\operatorname{deg}_{q} a_{(V, \boldsymbol{B})}^{J}}
$$

and a family of rational functions $\mathbf{F}=\left(F_{J}(\mathbf{X})\right)_{J \subseteq[n-1]}$ with the inversion property (IP) we define, similarly as above, a rational function

$$
\operatorname{Ig}_{(V, \boldsymbol{B})}\left(q^{-1}, \mathbf{X}\right):=\operatorname{Ig}_{(V, \boldsymbol{B}), \mathbf{F}}\left(q^{-1}, \mathbf{X}\right)=\sum_{J \subseteq[n-1]} \alpha_{(V, \boldsymbol{B})}^{J}\left(q^{-1}\right) F_{J}(\mathbf{X}) .
$$

The second main result of this paper is

Theorem B. For each $n$-dimensional vector space $V$, equipped with a flag of alternating bilinear (respectively Hermitian) forms $\boldsymbol{B}$ of type $I=\left\{i_{1}, \ldots, i_{r}\right\}<\subseteq[n-1]$, the associated Igusa-type function satisfies the functional equation

$$
\operatorname{Ig}_{(V, \boldsymbol{B})}\left(q, \mathbf{X}^{-1}\right)=(-1)^{a} q^{b} \operatorname{Ig}_{(V, \widetilde{\boldsymbol{B}})}\left(q^{-1}, \mathbf{X}\right),
$$

where $\widetilde{\boldsymbol{B}}$ is a flag of forms of type $\widetilde{I}:=\{n-i \mid i \in I\}$ and the integers $a$ and $b$ are given by Table 3 (with $m:=\left\lfloor\frac{n}{2}\right\rfloor$ ).

Note that, for $I=\varnothing$, Theorem $\mathrm{B}$ specialises to Theorem $\mathrm{A}$ in the symplectic and unitary cases, respectively. 
TABLE 3

\begin{tabular}{|c|c|c|}
\hline geometric type & $a$ & $b$ \\
\hline symplectic & $m-1$ & $m(m-1)+\left(\left(i_{2}-i_{1}\right) i_{1}+\cdots+\left(n-i_{r}\right) i_{r}\right) / 2$ \\
unitary & $n-1+b$ & $\left(\begin{array}{c}n \\
2\end{array}\right)+\left(i_{2}-i_{1}\right) i_{1}+\cdots+\left(n-i_{r}\right) i_{r}$ \\
\hline
\end{tabular}

To prove Theorem $\mathrm{B}$ we show that the functions $\alpha_{(V, \boldsymbol{B})}^{J}\left(q^{-1}\right)$ are polynomials which may be described in terms of a certain statistic on the Coxeter group $W$ of type $A_{\gamma n-1}$. This statistic associates to an element $w \in W$ the sum of its ordinary length $l(w)$ with respect to the standard Coxeter generating set $S=\left\{s_{1}, \ldots, s_{\gamma n-1}\right\}$ and its 'parabolic length' $l_{\mathrm{L}}^{(\gamma \widetilde{I})^{c}}(w)$. The parabolic length $l_{\mathrm{L}}^{(\gamma \widetilde{I})^{c}}(w)$ is the Coxeter length of the distinguished representative of shortest length in the left coset $w W_{(\gamma \widetilde{I})^{c}}$ of the standard parabolic subgroup $W_{(\gamma \widetilde{I})^{c}}=\left\langle s_{i} \in S \mid \gamma n-i \notin \gamma I\right\rangle$.

In fact, in Section 3 we show that Theorem B can be deduced from Theorem 2.6. a general result on rational functions defined in terms of linear combinations of parabolic length functions and characters on certain subgroups of finite Coxeter groups. Indeed, Theorem 2.6 extends to a slightly more general setting Igusa's key idea to deduce functional equations from features of the map induced by (right-) multiplication by the longest element.

Our initial interest in the Igusa-type functions $\operatorname{Ig}_{(V, \boldsymbol{B})}$ arose from our study of the zeta functions counting subgroups of higher Heisenberg groups. In 12 we introduce an equivalence relation, coarser than homothety, on the set of complete $\mathbb{Z}_{p}$-lattices in a non-degenerate symplectic $p$-adic vector space $\mathbb{Q}_{p}^{2 m}$ such that equivalence classes of lattices are in one-to-one correspondence with the vertices of the affine Bruhat-Tits building for the symplectic group $\operatorname{Sp}_{2 m}\left(\mathbb{Q}_{p}\right)$. For a flag of forms $\boldsymbol{B}$ of type $I=\{2 i\}, i \in[m]$, and suitable choices of $\mathbf{F}$ the Igusa functions $\operatorname{Ig}_{\left(\mathbb{F}_{q}^{2 m}, \boldsymbol{B}\right), \mathbf{F}}$ may be regarded as generating functions, enumerating lattices in an equivalence class indexed by a special vertex of type $i$. We refer to [12] for details.

1.2.3. Conjecture [C. In the last part of the paper we formulate a precise conjecture describing the polynomials $\alpha_{\mathcal{V}}^{J}\left(q^{-1}\right)$ in the orthogonal case. If it holds, the orthogonal case of Theorem $\mathrm{A}$ also follows from Theorem 2.6. Moreover, a proof of Conjecture $\mathrm{C}$ would constitute a first step towards extending Theorem $\mathrm{B}$ to the orthogonal case.

We introduce the subgroup $\mathcal{C}_{n}$ of 'chessboard elements' in the symmetric group $\mathcal{S}_{n}$ on $n$ letters. A permutation is a chessboard element if the non-zero entries of its associated permutation matrix all fit either on the black or on the white squares of an $n \times n$ chessboard. In Section 5 we define linear characters $\chi_{\varepsilon}$ on $\mathcal{C}_{n}$ and a certain linear combination $L$ of parabolic length functions on $\mathcal{S}_{n}$. By $D_{\mathrm{L}}(w)$ we denote the left-descent set of the permutation $w$ (cf. Section 2).

Conjecture C. For each n-dimensional, non-degenerate quadratic space $\mathcal{V}$ and each $J \subseteq[n-1]$,

$$
\alpha_{\mathcal{V}}^{J}\left(q^{-1}\right)=\sum_{\substack{w \in \mathcal{C}_{n} \\ D_{\mathrm{L}}(w) \subseteq J}} \chi_{\varepsilon}(w) q^{-L(w)}
$$

1.3. Organisation and notation. The structure of the paper is as follows. In Section 2 we derive functional equations for rational functions defined in terms of parabolic length functions on Coxeter groups. Theorem [2.6, the main result of 
Section 2 is applied to prove Theorem $\mathrm{B}$ in Section 3 . In Section 4 we prove the orthogonal case of Theorem A. In Section 5 we give a more precise statement of Conjecture C] Some explicit examples of Igusa-type functions can be found in the Appendix.

We use the following notation:

\begin{tabular}{|c|c|}
\hline & \\
\hline $\mathbb{N}$ & the set $\{1,2, \ldots\}$ of natural numbers \\
\hline$a, b]$ & the interval $\{a, a+1, \ldots, b\}$ for integers $a, b$ \\
\hline a] & the interval $[1, a]$ for an integer $a$ \\
\hline$\left.i_{1}, \ldots, i_{r}\right\}_{<}$ & the set $\left\{i_{1}, \ldots, i_{r}\right\} \subseteq \mathbb{N}_{0}$ with $i_{1}<\cdots<i_{r}$ \\
\hline 1 & $\begin{array}{l}\text { the set }[n-1] \backslash I \text { for } I \subseteq[n-1] \\
\quad \text { where } n \text { is clear from the context }\end{array}$ \\
\hline & $\begin{array}{l}\text { the set }\{n-i \mid i \in I\} \text { for } I \subseteq[n-1] \text {, } \\
\text { where } n \text { is clear from the context }\end{array}$ \\
\hline- & $\begin{array}{l}\text { the set }\left\{i_{1}-t_{1}, \ldots, i_{r}-t_{r}\right\} \cap \mathbb{N} \text { for } I=\left\{i_{1}, \ldots, i_{r}\right\}<\subseteq \mathbb{N} \\
\text { and } \mathbf{t}=\left(t_{1}, \ldots, t_{r}\right) \in \mathbb{N}_{0}^{r}\end{array}$ \\
\hline & $\begin{array}{l}\text { the set }\{i-j \mid i \in I\} \cap \mathbb{N} \text { for } I \subseteq \mathbb{N}, j \in \mathbb{N}_{0} \\
\text { the ordinary binomial coefficient for } a, b \in \mathbb{N}_{0}\end{array}$ \\
\hline$\left(\begin{array}{l}a \\
b\end{array}\right)$ & $\begin{array}{l}\text { the polynomial } \prod_{i=0}^{b-1}\left(1-X^{a-i}\right) /\left(1-X^{b-i}\right) \\
\quad \text { where } a, b \in \mathbb{N}_{0} \text { with } a \geq b\end{array}$ \\
\hline$\left(\begin{array}{l}n \\
J\end{array}\right)_{X}$ & $\begin{array}{l}\text { Note: The } q \text {-binomial coefficient }\left(\begin{array}{l}a \\
b\end{array}\right)_{q} \text { gives } \\
\text { the number of subspaces of dimension } b \text { in } \mathbb{F}_{q}^{a} \text {. } \\
\text { the polynomial }\left(\begin{array}{c}n \\
j_{s}\end{array}\right)_{X}\left(\begin{array}{c}j_{s} \\
j_{s-1}\end{array}\right)_{X} \ldots\left(\begin{array}{c}j_{2} \\
j_{1}\end{array}\right)_{X} \text {, } \\
\text { where } J=\left\{j_{1}, \ldots, j_{s}\right\}_{<} \subseteq[n-1]_{0} \text { for } n \in \mathbb{N}\end{array}$ \\
\hline $\begin{array}{l}x\rfloor \\
P(S)\end{array}$ & $\begin{array}{l}\text { Note: }\left(\begin{array}{l}n \\
J\end{array}\right)_{q} \text { gives the number of flags of type } J \backslash\{0\} \text { in } \mathbb{F}_{q}^{n} \text {. } \\
\text { the greatest integer not exceeding the rational number } x \\
\text { the power set of a set } S \\
\text { the symmetric group on } n \text { letters. }\end{array}$ \\
\hline & sup on $n$ letter \\
\hline
\end{tabular}

Throughout this paper, $n \in \mathbb{N}$ and $m=\lfloor n / 2\rfloor$. We shall write

$$
\begin{aligned}
I & =\left\{i_{1}, \ldots, i_{r}\right\}_{<}, \quad J=\left\{j_{1}, \ldots, j_{s}\right\}_{<} & & \text {for subsets of }[n-1] \text { or }[n], \text { and } \\
G & =\left\{g_{1}, \ldots, g_{k}\right\}_{<}, \quad H & & \text { for subsets of }[m] .
\end{aligned}
$$

\section{Rational functions From CoXeter groups}

In this section we prove functional equations for a family of rational functions associated to finite Coxeter systems. Theorem B will turn out to be a consequence of Theorem 2.6. the main result of the current section.

Let $(W, S)$ be a finite Coxeter system of rank $n-1$ with root system $\Delta$. To ease notation we will frequently identify the set of Coxeter generators $S=\left\{s_{1}, \ldots, s_{n-1}\right\}$ with the set of integers $[n-1]$. For each $I \subseteq S$ we denote by $W_{I}$ the corresponding standard parabolic subgroup of $W$ generated by the elements in $I$ and by $\Delta_{I}$ the induced root system. We denote by $l$ the length function on $W$ with respect to $S$. The length of an element $w$ may either be interpreted as the length of a shortest word in the elements of $S$ representing the group element or as the number of positive roots that are sent to negative roots by $w$. The group $W$ has a unique 
longest element $w_{0}$, whose length equals $|\Delta| / 2$. It is well known (cf. [10, Section 1.8]) that, for each $w \in W$,

$$
l\left(w_{0} w\right)+l(w)=l\left(w w_{0}\right)+l(w)=l\left(w_{0}\right) .
$$

The rational functions studied in this section are defined in terms of more general length functions. For each $I \subseteq S$ set

$$
\begin{aligned}
& W_{\mathrm{L}}^{I}:=\{w \in W \mid \forall s \in I: l(w s)>l(w)\}, \\
& W_{\mathrm{R}}^{I}:=\{w \in W \mid \forall s \in I: l(s w)>l(w)\} .
\end{aligned}
$$

We will need the following lemma ([15, Proposition 2.1.7]).

Lemma 2.1. Let $I \subseteq S$. Then $W_{\mathrm{L}}^{I}$ (respectively $W_{\mathrm{R}}^{I}$ ) is a left (respectively right) transversal to $W_{I}$ in $W$; i.e. for every $w \in W$ there are unique elements

$$
u_{\mathrm{L}} \in W_{\mathrm{L}}^{I}, v_{\mathrm{L}} \in W_{I} \text { and } u_{\mathrm{R}} \in W_{\mathrm{R}}^{I}, v_{\mathrm{R}} \in W_{I}
$$

such that

$$
w=u_{\mathrm{L}} v_{\mathrm{L}}=v_{\mathrm{R}} u_{\mathrm{R}}
$$

In particular, $u_{\mathrm{L}}$ is the unique element of shortest length in the left coset $w W_{I}$ and $u_{\mathrm{R}}$ is the unique element of shortest length in the right coset $W_{I} w$. Moreover,

$$
l(w)=l\left(u_{\mathrm{L}}\right)+l\left(v_{\mathrm{L}}\right)=l\left(v_{\mathrm{R}}\right)+l\left(u_{\mathrm{R}}\right) .
$$

The elements $u_{\mathrm{L}} \in w W_{I}$ and $u_{\mathrm{R}} \in W_{I} w$ are also characterised by the fact that they send positive roots of $\Delta_{I}$ to positive roots.

Definition 2.2 (Parabolic length). For each $I \subseteq S$ and $w \in W$ we set

$$
\begin{aligned}
l_{\mathrm{L}}^{I}(w) & :=l\left(u_{\mathrm{L}}\right), \\
l_{\mathrm{R}}^{I}(w) & :=l\left(u_{\mathrm{R}}\right) .
\end{aligned}
$$

We call $l_{\mathrm{L}}^{I}$ (respectively $l_{\mathrm{R}}^{I}$ ) the left (respectively right) parabolic length function on $W$ associated to $I$. We write $\mathbf{l}_{L}:=\left(l_{\mathrm{L}}^{I}\right)_{I \subseteq S}$ and $\mathbf{l}_{\mathrm{R}}:=\left(l_{\mathrm{R}}^{I}\right)_{I \subseteq S}$.

Note that for $I=\varnothing$ the corresponding parabolic length functions reduce to the ordinary Coxeter length function: $l_{\mathrm{L}}^{\varnothing}=l_{\mathrm{R}}^{\varnothing}=l$. Moreover, $l_{\mathrm{L}}^{S}=l_{\mathrm{R}}^{S}=0$.

Lemma 2.3. For each $I \subseteq S$ and $w \in W$ we have

$$
\begin{aligned}
l_{\mathrm{L}}^{I}\left(w_{0} w\right)+l_{\mathrm{L}}^{I}(w)=l_{\mathrm{L}}^{I}\left(w_{0}\right), & l_{\mathrm{L}}^{I}\left(w w_{0}\right)+l_{\mathrm{L}}^{I_{0}}(w)=l_{\mathrm{L}}^{I}\left(w_{0}\right), \\
l_{\mathrm{R}}^{I}\left(w_{0} w\right)+l_{\mathrm{R}}^{I w_{0}}(w)=l_{\mathrm{R}}^{I}\left(w_{0}\right), & l_{\mathrm{R}}^{I}\left(w w_{0}\right)+l_{\mathrm{R}}^{I}(w)=l_{\mathrm{R}}^{I}\left(w_{0}\right) .
\end{aligned}
$$

Proof. Let $v_{0}$ denote the longest element in $W_{I}$. Then

$$
l_{\mathrm{L}}^{I}\left(w_{0}\right)=l\left(w_{0}\right)-l\left(v_{0}\right)=l\left(w_{0}\right)-\left|\Delta_{I}\right| / 2 .
$$

Write $w=u_{\mathrm{L}} v_{\mathrm{L}}$ as in Lemma 2.1. We may then write

$$
w_{0}=u^{\prime} v^{\prime} v_{\mathrm{L}}^{-1} u_{\mathrm{L}}^{-1}
$$

with $u^{\prime} \in W, v^{\prime} \in W_{I}$ such that $v^{\prime} v_{\mathrm{L}}^{-1}=v_{0}$. It follows that

$$
\begin{aligned}
l\left(u^{\prime}\right) & =l\left(w_{0}\right)-l\left(v^{\prime} v_{\mathrm{L}}^{-1} u_{\mathrm{L}}^{-1}\right)=l\left(w_{0}\right)-l\left(u_{\mathrm{L}} v_{0}\right) \\
& =l\left(w_{0}\right)-l\left(u_{\mathrm{L}}\right)-l\left(v_{0}\right)=\left(l\left(w_{0}\right)-l\left(v_{0}\right)\right)-l\left(u_{\mathrm{L}}\right) \\
& =l_{\mathrm{L}}^{I}\left(w_{0}\right)-l_{\mathrm{L}}^{I}(w) .
\end{aligned}
$$


Clearly, $w_{0} w W_{I}=u^{\prime} W_{I}$. But $u^{\prime}$ sends positive roots of $\Delta_{I}$ to positive roots and is thus the unique coset representative of shortest length. Hence $l_{\mathrm{L}}^{I}\left(w_{0} w\right)=l\left(u^{\prime}\right)=$ $l_{\mathrm{L}}^{I}\left(w_{0}\right)-l_{\mathrm{L}}^{I}(w)$. This gives the first equation in (2.1).

For the second equation in (2.1), note that conjugation by $w_{0}$ yields $l_{\mathrm{L}}^{I}\left(w_{0}\right)=$ $l_{\mathrm{L}}^{I^{w_{0}}}\left(w_{0}\right)$ and thus

$$
l_{\mathrm{L}}^{I}\left(w w_{0}\right)=l_{\mathrm{L}}^{I_{0}}\left(w_{0} w\right)=l_{\mathrm{L}}^{I_{0}}\left(w_{0}\right)-l_{\mathrm{L}}^{I^{w_{0}}}(w)=l_{\mathrm{L}}^{I}\left(w_{0}\right)-l_{\mathrm{L}}^{I^{w_{0}}}(w) .
$$

We omit the analogous proofs for the equations (2.2).

Another important invariant of an element of a Coxeter group which we shall need is its (left) descent set $D_{\mathrm{L}}(w):=\{s \in S \mid l(s w)<l(w)\}$. Note that

$$
D_{\mathrm{L}}\left(w w_{0}\right)=D_{\mathrm{L}}(w)^{c}:=\left\{s \in S \mid s \notin D_{\mathrm{L}}(w)\right\} .
$$

Elements of Coxeter groups of type $A$ can be regarded as permutation matrices. It is noteworthy that both the descent sets and the values of the various parabolic length functions are easily read off from the associated matrices.

Lemma 2.4. Let $\left(F_{J}(\mathbf{X})\right)_{J \subseteq S}$ be a family of rational functions with the inversion property (IP). Then, for all $I \subseteq S$,

$$
\sum_{I \subseteq J \subseteq S} F_{J}\left(\mathbf{X}^{-1}\right)=(-1)^{|S|} \sum_{I^{c} \subseteq J \subseteq S} F_{J}(\mathbf{X}) .
$$

Proof. This is an easy calculation. See [19, Lemma 7].

We now fix a family of rational functions $\mathbf{F}=\left(F_{J}(\mathbf{X})\right)_{J \subseteq S}$ with the inversion property (IP) and an independent indeterminate $Y$. We choose a family $\mathbf{b}=$ $\left(b_{I}\right)_{I \subseteq S}$ of integers and define the statistics $\mathbf{b} \cdot \mathbf{l}_{\mathrm{L}}$ and $\mathbf{b} \cdot \mathbf{l}_{\mathrm{R}}$ on $W$ by setting, for $w \in W$

$$
\mathbf{b} \cdot \mathbf{l}_{\mathrm{L}}(w):=\sum_{I \subseteq S} b_{I} l_{\mathrm{L}}^{I}(w), \quad \mathbf{b} \cdot \mathbf{l}_{\mathrm{R}}(w):=\sum_{I \subseteq S} b_{I} l_{\mathrm{R}}^{I}(w) .
$$

Similarly, we write $\mathbf{b}^{w_{0}} \cdot \mathbf{l}_{\mathrm{L}}$ and $\mathbf{b}^{w_{0}} \cdot \mathbf{l}_{\mathrm{R}}$ to denote the statistics associating to $w$ the elements $\sum_{I \subseteq S} b_{I} l_{\mathrm{L}}^{I_{0} w_{0}}(w)$ and $\sum_{I \subseteq S} b_{I} l_{\mathrm{R}}^{I^{w_{0}}}(w)$, respectively. Let $W^{\prime} \subseteq W$ be a subgroup with $w_{0} \in W^{\prime}$, and $\chi: W^{\prime} \rightarrow \mathbb{C}^{*}$ a (linear) character of $W^{\prime}$.

Definition 2.5. With the given data we define the following rational functions:

$$
\begin{aligned}
\mathrm{IG}_{\mathrm{L}}^{W^{\prime}, \mathbf{b}, \chi, \mathbf{F}}(Y, \mathbf{X}) & :=\sum_{w \in W^{\prime}} \chi(w) Y^{\mathbf{b} \cdot \mathrm{l}_{\mathrm{L}}(w)} \sum_{D_{\mathrm{L}}(w) \subseteq J \subseteq S} F_{J}(\mathbf{X}), \\
\mathrm{IG}_{\mathrm{R}}^{W^{\prime}, \mathbf{b}, \chi, \mathbf{F}}(Y, \mathbf{X}) & :=\sum_{w \in W^{\prime}} \chi(w) Y^{\mathbf{b} \cdot \mathbf{l}_{\mathrm{R}}(w)} \sum_{D_{\mathrm{L}}(w) \subseteq J \subseteq S} F_{J}(\mathbf{X}) .
\end{aligned}
$$

The main result of the current section is

Theorem 2.6. The following functional equations hold:

$$
\begin{aligned}
& \mathrm{IG}_{\mathrm{L}}^{W^{\prime}, \mathbf{b}, \chi, \mathbf{F}}\left(Y^{-1}, \mathbf{X}^{-1}\right)=(-1)^{|S|} \chi\left(w_{0}\right) Y^{-\mathbf{b} \cdot \mathbf{l}_{\mathrm{L}}\left(w_{0}\right)} \mathrm{IG}_{\mathrm{L}}^{W^{\prime}, \mathbf{b}^{w_{0}}, \chi, \mathbf{F}}(Y, \mathbf{X}), \\
& \mathrm{IG}_{\mathrm{R}}^{W^{\prime}, \mathbf{b}, \chi, \mathbf{F}}\left(Y^{-1}, \mathbf{X}^{-1}\right)=(-1)^{|S|} \chi\left(w_{0}\right) Y^{-\mathbf{b} \cdot \mathbf{l}_{\mathrm{R}}\left(w_{0}\right)} \operatorname{IG}_{\mathrm{R}}^{W^{\prime}, \mathbf{b}, \chi, \mathbf{F}}(Y, \mathbf{X}) .
\end{aligned}
$$

Proof. The equations

$$
\begin{aligned}
\mathbf{b}^{w_{0}} \cdot \mathbf{l}_{\mathrm{L}}\left(w w_{0}\right)+\mathbf{b} \cdot \mathbf{l}_{\mathrm{L}}(w) & =\mathbf{b} \cdot \mathbf{l}_{\mathrm{L}}\left(w_{0}\right), \\
\mathbf{b} \cdot \mathbf{l}_{\mathrm{R}}\left(w w_{0}\right)+\mathbf{b} \cdot \mathbf{l}_{\mathrm{R}}(w) & =\mathbf{b} \cdot \mathbf{l}_{\mathrm{R}}\left(w_{0}\right)
\end{aligned}
$$


are immediate consequences of Lemma 2.3 . Therefore, by (2.6), by Lemma 2.4 and by (2.3),

$$
\begin{aligned}
& \mathrm{IG}_{\mathrm{L}}^{W^{\prime}, \mathbf{b}, \chi, \mathbf{F}}\left(Y^{-1}, \mathbf{X}^{-1}\right)=\sum_{w \in W^{\prime}} \chi(w) Y^{-\mathbf{b} \cdot \mathbf{l}_{\mathrm{L}}(w)} \sum_{D_{\mathrm{L}}(w) \subseteq J \subseteq S} F_{J}\left(\mathbf{X}^{-1}\right) \\
& =(-1)^{|S|} \chi\left(w_{0}\right)^{-1} Y^{-\mathbf{b} \cdot \mathbf{l}_{\mathrm{L}}\left(w_{0}\right)} \sum_{w \in W^{\prime}} \chi\left(w w_{0}\right) Y^{\mathbf{b}^{w_{0}} \cdot \mathbf{l}_{\mathrm{L}}\left(w w_{0}\right)} \sum_{D_{\mathrm{L}}\left(w w_{0}\right) \subseteq J \subseteq S} F_{J}(\mathbf{X}) \\
& =(-1)^{|S|} \chi\left(w_{0}\right) Y^{-\mathbf{b} \cdot \mathbf{l}_{\mathrm{L}}\left(w_{0}\right)} \mathrm{IG}_{\mathrm{L}}^{W^{\prime}, \mathbf{b}^{w_{0}}, \chi, \mathbf{F}}(Y, \mathbf{X}) .
\end{aligned}
$$

The equation (2.5) is proved analogously.

In this paper we shall see instances of both types of functional equations presented in Theorem 2.6. In Section 3 we demonstrate that Theorem $\mathrm{B}$ is a consequence of (2.4). Note that in the special case $\mathbf{F}=\left(\prod_{j \in J} \frac{X_{j}}{1-X_{j}}\right)_{J \subseteq[n-1]}$, replacing $\mathbf{b}$ by $\mathbf{b}^{w_{0}}$ in (2.4) simply amounts to inverting the order of the variables $X_{1}, \ldots, X_{n-1}$. If Conjecture $[\mathrm{C}$ holds, the orthogonal case of Theorem $\mathrm{A}$ follows from (2.5).

\section{The SYMPLECTIC AND UNITARY CASES}

In this section we study the polynomials enumerating flags which are non-degenerate with respect to a 'flag of sesquilinear forms'. Our aim is to prove Theorem B, Let $V$ be an $n$-dimensional vector space over a field $F$. Let $I=\left\{i_{1}, \ldots, i_{r}\right\}_{<} \subseteq$ $[n-1]$, and set $i_{0}:=0, i_{r+1}:=n$.

Definition 3.1 (Flag of forms). We say that $V$ is equipped with a flag of alternating bilinear (respectively Hermitian) forms $\boldsymbol{B}=\left(B_{i_{1}}, \ldots, B_{i_{r+1}}\right)$ of type $I$ if there is a filtration of subspaces

$$
\{0\}=: R_{i_{0}} \subset R_{i_{1}} \subset \cdots \subset R_{i_{r}} \subset R_{i_{r+1}}:=V
$$

such that

(a) for all $i \in I, \operatorname{dim} R_{i}=i$;

(b) for all $\rho \in[r+1], B_{i_{\rho}}$ is an alternating bilinear (respectively Hermitian) form $B_{i_{\rho}}: R_{i_{\rho}} \times R_{i_{\rho}} \rightarrow F$ with

$$
\operatorname{Rad}\left(B_{i_{\rho}}\right):=\left\{x \in R_{i_{\rho}} \mid \forall y \in R_{i_{\rho}}: B_{i_{\rho}}(x, y)=0\right\}=R_{i_{\rho-1}} .
$$

We call the sequence $\mathbf{R}=\left(R_{i_{1}}, \ldots, R_{i_{r}}\right)$ the flag of radicals associated to the flag of forms $\boldsymbol{B}$.

Note that, given a flag of sesquilinear forms $\boldsymbol{B}$ of type $I$ on $V$ with flag of radicals $\mathbf{R}$ and $\rho \in[r+1]$, we have a flag of forms $\left(B_{i_{1}}, \ldots, B_{i_{\rho}}\right)$ of type $\left\{i_{1}, \ldots, i_{\rho-1}\right\}$ on $R_{i_{\rho}}$ with flag of radicals $\left(R_{i_{1}}, \ldots, R_{i_{\rho-1}}\right)$ and a flag of forms $\left(\overline{B_{i_{\rho+1}}}, \ldots, \overline{B_{i_{r+1}}}\right)$ of type $\left\{i_{\varrho}-i_{\rho} \mid \rho<\varrho \leq r\right\}$ on $V / R_{i_{\rho}}$ with flag of radicals $\left(R_{i_{\rho+1}} / R_{i_{\rho}}, \ldots, R_{i_{r}} / R_{i_{\rho}}\right)$.

Definition 3.2 (Non-degeneracy). Given a flag of sesquilinear forms $\boldsymbol{B}$ of type $I$ on $V$ with flag of radicals $\mathbf{R}$ as above, we say that a subspace $U \subseteq V$ is nondegenerate with respect to $\boldsymbol{B}$ if for each $\rho \in[r+1]$,

(a) $U \cap R_{i_{\rho}}$ is non-degenerate with respect to $\left(B_{i_{1}}, \ldots, B_{i_{\rho}}\right)$ and

(b) $\left(U+R_{i_{\rho}}\right) / R_{i_{\rho}}$ is non-degenerate with respect to $\left(\overline{B_{i_{\rho+1}}}, \ldots, \overline{B_{i_{r+1}}}\right)$.

A flag $\mathbf{U}_{J}=\left(U_{j}\right)_{j \in J}$ of subspaces of $V$ of type $J \subseteq[n-1]$, i.e. an ascending chain of subspaces with $\operatorname{dim} U_{j}=j$ for each $j \in J$, is said to be non-degenerate with respect to $\boldsymbol{B}$ if each of its constituents $U_{j}$ is. 
These definitions are illustrated by the following simple example.

Example 3.3. Suppose that $n$ is even, $r=1$ and $i_{1} \in[n-1]$ is even. Then a flag of alternating bilinear forms $\boldsymbol{B}=\left(B_{i_{1}}, B_{n}\right)$ consists of a (degenerate) alternating bilinear form $B_{n}$ on $V$ with $i_{1}$-dimensional radical $R_{i_{1}}$, which in turn supports a non-degenerate form $B_{i_{1}}$. A subspace $U \subseteq V$ is non-degenerate with respect to $\boldsymbol{B}=\left(B_{i_{1}}, B_{n}\right)$ if $U \cap R_{i_{1}}$ is non-degenerate with respect to $B_{i_{1}}$ and $\left(U+R_{i_{1}}\right) / R_{i_{1}}$ is non-degenerate with respect to $\overline{B_{n}}$.

We shall now assume that $V$ is an $n$-dimensional vector space over a finite field $F$, equipped with a flag of sesquilinear forms $\boldsymbol{B}$ of type $I$. As in the introduction, we write $\gamma=1 / 2$ in the symplectic case and $\gamma=1$ in the unitary case so that $F=\mathbb{F}_{q^{2 \gamma}}$ for some prime power $q$. Let $J \subseteq[n-1]$ and define $a_{(V, \boldsymbol{B})}^{J}(q)$ to be the number of flags of type $J$ which are non-degenerate with respect to $\boldsymbol{B}$. We set

$$
\alpha_{(V, \boldsymbol{B})}^{J}\left(q^{-1}\right):=a_{(V, \boldsymbol{B})}^{J}(q) / q^{\operatorname{deg}_{q} a_{(V, \boldsymbol{B})}^{J}}
$$

and shall frequently write $a_{n, I}^{J}(q)$ for $a_{(V, \boldsymbol{B})}^{J}(q)$ and $\alpha_{n, I}^{J}\left(q^{-1}\right)$ for $\alpha_{(V, \boldsymbol{B})}^{J}\left(q^{-1}\right)$. Recall that, in the symplectic case, both the type $I$ of a flag of forms and all the sets $J \subseteq[n-1]$ for which $a_{(V, \boldsymbol{B})}^{J}(q)$ is non-zero consist necessarily of even numbers.

Definition 3.4. Given a family $\mathbf{F}=\left(F_{J}(\mathbf{X})\right)_{J \subseteq[n-1]}$ of rational functions with the inversion property (IP) we define

$$
\operatorname{Ig}_{(V, \boldsymbol{B})}\left(q^{-1}, \mathbf{X}\right):=\operatorname{Ig}_{(V, \boldsymbol{B}), \mathbf{F}}\left(q^{-1}, \mathbf{X}\right)=\sum_{J \subseteq[n-1]} \alpha_{(V, \boldsymbol{B})}^{J}\left(q^{-1}\right) F_{J}(\mathbf{X}) .
$$

Theorem B states that these Igusa-type functions satisfy a functional equation. In the remainder of the current section we show how this can be deduced from the first assertion of Theorem 2.6. Fix a family of rational functions $\mathbf{F}=$ $\left(F_{J}(\mathbf{X})\right)_{J \subseteq[n-1]}$ with the inversion property (IP) and define

$$
\gamma \mathbf{F}:=\left(F_{\gamma^{-1} J^{\prime}}(\mathbf{X})\right)_{J^{\prime} \subseteq[\gamma n-1]} .
$$

Let $W^{\prime}:=W:=\mathcal{S}_{\gamma n}$ be the full symmetric group on $\gamma n$ letters, let $\chi$ be the trivial character on $W^{\prime}$ and set, for each $J^{\prime} \subseteq[\gamma n-1]$,

$$
b_{J^{\prime}}:=\delta\left({ }^{\prime} J^{\prime}=\varnothing^{\prime}\right)+\delta\left({ }^{\prime} J^{\prime}=\left\{s_{i} \mid i \notin \gamma \widetilde{I}\right\}^{\prime}\right),
$$

where the Kronecker delta $\delta(E) \in\{1,0\}$ reflects whether or not equation $E$ holds.

In order to derive Theorem B from (2.4) it suffices to show that

$$
\operatorname{Ig}_{(V, \boldsymbol{B}), \mathbf{F}}\left(q^{-1}, \mathbf{X}\right)=\operatorname{IG}_{\mathrm{L}}^{W^{\prime}, \mathbf{b}, \chi, \gamma \mathbf{F}}\left((-q)^{-1 / \gamma}, \mathbf{X}\right)
$$

for the given data $W^{\prime}, \mathbf{b}, \chi$ and $\gamma \mathbf{F}$. Clearly, it is enough to prove

Proposition 3.5. Let $J \subseteq[n-1]$ such that $\gamma J \subseteq[\gamma n-1]$. Then

$$
\alpha_{n, I}^{J}\left(q^{-1}\right)=a_{n, I}^{J}(q) / q^{2 \gamma \operatorname{deg}_{q}\left(\begin{array}{l}
n \\
J
\end{array}\right)_{q}}=\sum_{\substack{w \in \mathcal{S}_{\gamma n} \\
D_{\mathrm{L}}(w) \subseteq \subseteq_{\gamma}}} Y^{\lambda_{n, I}(w)}
$$

where $Y:=(-q)^{-1 / \gamma}$ and $\lambda_{n, I}(w):=l(w)+l_{\mathrm{L}}^{(\gamma \widetilde{I})^{c}}(w)$ for all $w \in W$. 
Proof. We will first prove (3.1) in the case $I=\varnothing$. The proof consists of a simple index computation in the respective isometry group, i.e. in the symplectic group $\operatorname{Sp}_{n}\left(\mathbb{F}_{q}\right)$ or the unitary group $\mathrm{U}_{n}\left(\mathbb{F}_{q^{2}}\right)$. The proof in the general case is then based on a recursive expression for the numbers $a_{n, I}^{J}(q)$.

So assume that $I=\varnothing$. Then $\boldsymbol{B}=(B)$ simply specifies a non-degenerate alternating bilinear (respectively Hermitian) form on $V$. The respective isometry group $\mathrm{Sp}_{n}\left(\mathbb{F}_{q}\right)$ or $\mathrm{U}_{n}\left(\mathbb{F}_{q^{2}}\right)$ acts transitively on the non-degenerate flags of type $J$, so it suffices to compute the stabiliser of any one of them. We construct a 'standard' nondegenerate flag $\mathbf{U}_{J}:=\left(U_{j_{1}}, \ldots, U_{j_{s}}\right)$ of type $J=\left\{j_{1}, \ldots, j_{s}\right\}_{<}$in the following way. In the symplectic case, choose a symplectic basis $E=\left(e_{1}, f_{1}, \ldots, e_{n / 2}, f_{n / 2}\right)$ for $V$ (i.e. $\left.B\left(e_{i}, f_{j}\right)=\delta_{i j}, B\left(e_{i}, e_{j}\right)=B\left(f_{i}, f_{j}\right)=0\right)$ and set $U_{j}:=\left\langle e_{1}, f_{1}, \ldots, e_{j / 2}, f_{j / 2}\right\rangle$ for $j \in J$. In the unitary case, choose a unitary basis $E=\left(e_{1}, \ldots, e_{n}\right)$ for $V$ (i.e. $\left.B\left(e_{i}, e_{j}\right)=\delta_{i j}\right)$ and set $U_{j}:=\left\langle e_{1}, \ldots, e_{j}\right\rangle$ for $j \in J$. It is not difficult to verify that an element of the respective isometry group of $(V, B)$ stabilises $\mathbf{U}_{J}$ if and only if its matrix $M_{n}$ with respect to the basis $E$ is of block diagonal form

$$
M_{n}=\left(\begin{array}{cccc}
M_{j_{1}} & & & \\
& M_{j_{2}-j_{1}} & & \\
& & \ddots & \\
& & & M_{n-j_{s}}
\end{array}\right)
$$

with $M_{j_{\sigma}-j_{\sigma-1}}$ in the respective smaller isometry group for all $\sigma \in[s+1]$, where $j_{0}:=0, j_{s+1}:=n$. Thus

$$
a_{n, \varnothing}^{J}(q)= \begin{cases}\left|\mathrm{Sp}_{n}\left(\mathbb{F}_{q}\right)\right| / \prod_{\sigma \in[s+1]}\left|\mathrm{Sp}_{j_{\sigma}-j_{\sigma-1}}\left(\mathbb{F}_{q}\right)\right| & \text { in the symplectic case } \\ \left|\mathrm{U}_{n}\left(\mathbb{F}_{q^{2}}\right)\right| / \prod_{\sigma \in[s+1]}\left|\mathrm{U}_{j_{\sigma}-j_{\sigma-1}}\left(\mathbb{F}_{q^{2}}\right)\right| & \text { in the unitary case. }\end{cases}
$$

Employing the well-known formulae (cf. [1, p. 147], 9, Theorems 3.12 and 11.28])

$$
\begin{aligned}
& \left|\operatorname{Sp}_{n}\left(\mathbb{F}_{q}\right)\right|=q^{\left(\begin{array}{c}
n+1 \\
2
\end{array}\right)} \prod_{i \in[n / 2]}\left(1-q^{-2 i}\right), \\
& \left|\mathrm{U}_{n}\left(\mathbb{F}_{q^{2}}\right)\right|=q^{n^{2}} \prod_{i \in[n]}\left(1-\left(-q^{-1}\right)^{i}\right)
\end{aligned}
$$

and using the notation $Y=(-q)^{-1 / \gamma}$ we obtain

$$
\alpha_{n, \varnothing}^{J}\left(q^{-1}\right)=\frac{\prod_{i \in[\gamma n]}\left(1-Y^{i}\right)}{\prod_{\sigma \in[s+1]} \prod_{\iota \in\left[\gamma\left(j_{\sigma}-j_{\sigma-1}\right)\right]}\left(1-Y^{\iota}\right)}=\left(\begin{array}{c}
\gamma n \\
\gamma J
\end{array}\right)_{Y} .
$$

It is equally well known (cf. [16, Example 2.2.5]) that Gaussian polynomials may be expressed in terms of Coxeter length functions on symmetric groups:

$$
\left(\begin{array}{l}
\gamma n \\
\gamma J
\end{array}\right)_{Y}=\sum_{\substack{w \in \mathcal{S}_{\gamma n} \\
D_{\mathrm{L}}(w) \subseteq \gamma J}} Y^{l(w)}
$$

Equation (3.1) follows in the particular case $I=\varnothing$, as $l_{\mathrm{L}}^{(\gamma \widetilde{I})^{c}}=l_{\mathrm{L}}^{S}=0$ and $\lambda_{n, I}=l$.

We now treat the general case $I=\left\{i_{1}, \ldots, i_{r}\right\}_{<} \subseteq[n-1]$. To prove (3.1) we argue by induction on $n$. The base step $n=0$ is trivial, so suppose that $n>0$. We may further assume that $J=\left\{j_{1}, \ldots, j_{s}\right\}_{<} \neq \varnothing$ and we define $j:=j_{1}=\min J$. Our first aim is to derive a recursive formula for $a_{n, I}^{J}(q)$, using the formula we obtained in the special case $I=\varnothing$. For this purpose we determine what are the possible first 
terms $U_{j}$ of the flags $\mathbf{U}_{J}$ we intend to count. Then we consider in how many ways each such space $U_{j}$ can be completed to yield a full flag $\mathbf{U}_{J}$.

Let $T$ be the set of all $r$-tuples $\mathbf{t}=\left(t_{1}, \ldots, t_{r}\right) \in\left([j]_{0}\right)^{r}$ such that

$$
\begin{aligned}
& t_{1} \leq \cdots \leq t_{r}, \quad \gamma\left\{t_{1}, \ldots, t_{r}\right\} \subseteq[\gamma j]_{0} \quad \text { and } \\
& \forall \rho \in[r+1]: j-\left(n-i_{\rho}\right)-t_{\rho-1} \leq t_{\rho}-t_{\rho-1} \leq i_{\rho}-i_{\rho-1},
\end{aligned}
$$

where $i_{0}=t_{0}=0$ and $i_{r+1}:=n, t_{r+1}:=j$. These 'admissible' tuples encode the possible dimensions of the intersections $U_{j} \cap R_{i_{\rho}}$ of a $j$-dimensional subspace $U_{j}$ of $V$, non-degenerate with respect to $\boldsymbol{B}$, with the members $R_{i_{\rho}}$ of the flag of radicals associated to $B$. Recalling that the underlying field $F$ has cardinality $q^{2 \gamma}$ and applying (3.1) for $I=\varnothing$, we note that for each $\mathbf{t} \in T$ there are precisely

$$
\begin{aligned}
A_{n, I}^{\mathrm{t}}(q) & =\prod_{\rho \in[r+1]} a_{\left(i_{\rho}-i_{\rho-1}\right), \varnothing}^{\left\{t_{\rho}-t_{\rho-1}\right\}}\left(q^{-1}\right) q^{2 \gamma\left(t_{\rho}-t_{\rho-1}\right)\left(i_{\rho-1}-t_{\rho-1}\right)} \\
& =\prod_{\rho \in[r+1]} q^{2 \gamma\left(t_{\rho}-t_{\rho-1}\right)\left(i_{\rho}-i_{\rho-1}\right)} \prod_{\rho \in[r+1]} \sum_{\substack{w \in \mathcal{S}_{\gamma\left(i_{\rho}-i_{\rho-1}\right)} \\
D_{\mathrm{L}}(w) \subseteq \gamma\left\{t_{\rho}-t_{\rho-1}\right\}}} Y^{l(w)}
\end{aligned}
$$

subspaces $U_{j}$, non-degenerate with respect to $\boldsymbol{B}$, such that $\operatorname{dim}\left(U_{j} \cap R_{i_{\rho}}\right)=t_{\rho}$ for all $\rho \in[r+1]$. Given such a subspace $U_{j}$, the number of non-degenerate flags $\mathbf{U}_{J}$ with first term $U_{j}$ can be described inductively, using the notation $J-j=$ $\left\{j_{2}-j, \ldots, j_{s}-j\right\}$ and $I-\mathbf{t}=\left\{i_{1}-t_{1}, \ldots, i_{r}-t_{r}\right\} \cap \mathbb{N}$; it equals

$$
a_{n-j, I-\mathbf{t}}^{J-j}(q)=q^{2 \gamma \operatorname{deg}_{q}\left(\begin{array}{c}
n-j \\
J-j
\end{array}\right)_{q}} \sum_{\begin{array}{c}
w \in \mathcal{S}_{\gamma(n-j)} \\
D_{\mathrm{L}}(w) \subseteq \gamma(J-j)
\end{array}} Y^{\lambda_{n-j, I-\mathbf{t}}(w)} .
$$

For $\mathbf{t} \in T$, apply equations (3.3) and (3.4) together with the identities

$$
\sum_{\rho \in[r+1]}\left(t_{\rho}-t_{\rho-1}\right)\left(i_{\rho}-t_{\rho}\right)=j(n-j)-\sum_{\rho \in[r]} t_{\rho}\left(i_{\rho+1}-i_{\rho}-\left(t_{\rho+1}-t_{\rho}\right)\right)
$$

and

$$
\left(\begin{array}{l}
n \\
J
\end{array}\right)_{q}=\left(\begin{array}{l}
n \\
j
\end{array}\right)_{q}\left(\begin{array}{c}
n-j \\
J-j
\end{array}\right)_{q}, \quad \operatorname{deg}_{q}\left(\begin{array}{c}
n \\
j
\end{array}\right)_{q}=j(n-j)
$$

to obtain

$$
\begin{aligned}
\alpha_{n, I}^{J}\left(q^{-1}\right)= & a_{n, I}^{J}(q) / q^{2 \gamma \operatorname{deg}_{q}\left(\begin{array}{l}
n \\
J
\end{array}\right)_{q}} \\
= & q^{-2 \gamma \operatorname{deg}_{q}\left(\begin{array}{l}
n \\
J
\end{array}\right)} \sum_{\mathbf{t} \in T} A_{n, I}^{\mathbf{t}}(q) a_{n-j, I-\mathbf{t}}^{J-j}(q) \\
= & \sum_{\mathbf{t} \in T} Y^{2\left(\sum_{\rho \in[r]} \gamma t_{\rho}\left(\gamma\left(i_{\rho+1}-i_{\rho}\right)-\gamma\left(t_{\rho+1}-t_{\rho}\right)\right)\right)} \\
& \cdot\left(\sum_{\substack{w \in \mathcal{S}_{\gamma(n-j)} \\
D_{\mathrm{L}}(w) \subseteq \gamma(J-j)}} Y^{\lambda_{n-j, I-\mathbf{t}}(w)}\right)\left(\prod_{\rho \in[r+1]} \sum_{\substack{w \in \mathcal{S}_{\gamma\left(i_{\rho}-i_{\rho-1}\right)} \\
D_{\mathrm{L}}(w) \subseteq \gamma\left\{t_{\rho}-t_{\rho-1}\right\}}} Y^{l(w)}\right) .
\end{aligned}
$$

We are looking to prove that the right-hand side of equation (3.5) may be written as a sum over the elements in the symmetric group $\mathcal{S}_{\gamma n}$ whose left descent set is contained in $\gamma J$. In the following considerations we shall identify permutations $w \in \mathcal{S}_{\gamma n}$ (acting on $\{1, \ldots, \gamma n\}$ from the right) with the corresponding 
$\gamma n \times \gamma n$ permutation matrices (acting on the set of standard row vectors by rightmultiplication). Observe that for any element $w \in \mathcal{S}_{\gamma n}$ with $D_{\mathrm{L}}(w) \subseteq \gamma J$ the corresponding permutation matrix is ascending on the first $[\gamma j]$ rows. Define

$$
\mathbf{t}=\mathbf{t}(w)=\left(t_{1}, \ldots, t_{r}\right)
$$

by

$$
t_{\rho}:=\gamma^{-1}\left|\left\{\varrho \in[\gamma j] \mid \varrho^{w}>\gamma\left(n-i_{\rho}\right)\right\}\right| \text { for all } \rho \in[r],
$$

and set $t_{0}:=0, t_{r+1}:=j$. Then $\mathbf{t} \in T$, as $n-i_{\rho} \geq j-t_{\rho}$ for all $\rho \in[r+1]$ and thus $\mathbf{t}$ satisfies (3.2). Applying suitable elementary column operations to $w$ corresponding to left multiplication by elements of the parabolic subgroup $W_{(\gamma \widetilde{I})^{c}}$, it is easily seen that there are unique elements $u, v \in \mathcal{S}_{\gamma n}$ such that

(a) $w=u v$ and $l(w)=l(u)+l(v)$;

(b) for all $\rho \in[r+1]$ :

$$
\varrho \in\left[\gamma\left(j-t_{\rho}\right)+1, \gamma\left(j-t_{\rho-1}\right)\right] \Longleftrightarrow \varrho^{u}=\varrho+\gamma\left(n-i_{\rho}\right) ;
$$

(c) $v \in W_{(\gamma \widetilde{I})^{c}}$, i.e. for all $\rho \in[r+1]$ :

$$
\begin{aligned}
& \varrho \in\left[\gamma\left(n-i_{\rho}\right)+1, \gamma\left(n-i_{\rho-1}\right)\right] \Longleftrightarrow \varrho^{v} \in\left[\gamma\left(n-i_{\rho}\right)+1, \gamma\left(n-i_{\rho-1}\right)\right], \\
& \quad \text { and } D_{\mathrm{L}}(v) \subseteq\left\{\gamma\left(n-i_{\rho}\right)+\gamma\left(t_{\rho}-t_{\rho-1}\right) \mid \rho \in[r+1]\right\} .
\end{aligned}
$$

This is best seen in terms of permutation matrices. We write $\operatorname{id}_{s}$ for the $s \times s$ unit matrix. Then the permutation matrix $u$ has the shape

$$
\left(\begin{array}{l|l|l|l|l|l|l}
\mathrm{id}_{\gamma\left(j-t_{r}\right)} & & \ldots & & & & \\
\hline & & & & & & \\
\hline & & \ddots & & & & \\
\hline & & & \operatorname{id}_{\gamma\left(t_{2}-t_{1}\right)} & & & \\
\hline & & & & & \operatorname{id}_{\gamma t_{1}} & \\
\hline & u_{r+1} & \ldots & & u_{2} & & u_{1}
\end{array}\right),
$$

where $u_{\rho}$ is a $\gamma(n-j) \times \gamma\left(\left(i_{\rho}-i_{\rho-1}\right)-\left(t_{\rho}-t_{\rho-1}\right)\right)$ matrix for $\rho \in[r+1]$. The permutation matrix $v$ has the form

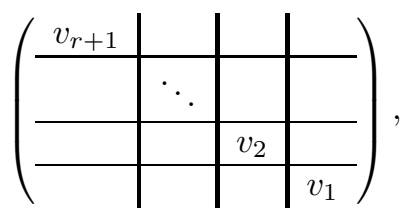

where $v_{\rho}$ is a $\gamma\left(i_{\rho}-i_{\rho-1}\right) \times \gamma\left(i_{\rho}-i_{\rho-1}\right)$ permutation matrix with at most one descent for $\rho \in[r+1]$. We may thus identify $v$ with

$$
\left(v_{1}, \ldots, v_{r+1}\right) \in \mathcal{S}_{\gamma i_{1}} \times \mathcal{S}_{\gamma\left(i_{2}-i_{1}\right)} \times \cdots \times \mathcal{S}_{\gamma\left(n-i_{r}\right)}
$$

and have, by slight abuse of notation, for each $\rho \in[r+1]$,

$$
D_{\mathrm{L}}\left(v_{\rho}\right) \subseteq\left\{\gamma\left(t_{\rho}-t_{\rho-1}\right)\right\} \cap\left[\gamma\left(i_{\rho}-i_{\rho-1}\right)-1\right] .
$$

Remark 3.6. The above decomposition $w=u v$ is not the one from Lemma 2.1. It is important for our purpose that each $v_{\rho}$ has at most one descent. 
Note that, by deleting the first $\gamma j$ rows and respective columns in (3.6), the element $u$ determines a unique $\gamma(n-j) \times \gamma(n-j)$ permutation matrix

$$
u^{\prime}:=\left(\begin{array}{llll}
u_{r+1} & \cdots & u_{2} & u_{1}
\end{array}\right)
$$

with descent set $D_{\mathrm{L}}\left(u^{\prime}\right)=D_{\mathrm{L}}(w)-\gamma j$.

As we indicated in Section 2, it is easy to determine the length of a permutation given by a permutation matrix: it is simply the number of entries 0 in the matrix which are not below or to the right of an entry 1 . Thus

$$
\begin{aligned}
& l(u)=\sum_{\rho \in[r]} \gamma t_{\rho}\left(\gamma\left(i_{\rho+1}-i_{\rho}\right)-\gamma\left(t_{\rho+1}-t_{\rho}\right)\right)+l\left(u^{\prime}\right), \\
& l(v)=\sum_{\rho \in[r+1]} l\left(v_{\rho}\right) .
\end{aligned}
$$

Moreover, the parabolic length of $w$ with respect to $(\gamma \widetilde{I})^{c}$ is determined by $\mathbf{t}$ and by the parabolic length of $u^{\prime}$ with respect to $(\gamma(\widetilde{I-\mathbf{t}}))^{c}$ :

$$
l_{\mathrm{L}}^{(\gamma \widetilde{I})^{c}}(w)=\sum_{\rho \in[r]} \gamma t_{\rho}\left(\gamma\left(i_{\rho+1}-i_{\rho}\right)-\gamma\left(t_{\rho+1}-t_{\rho}\right)\right)+l_{\mathrm{L}}^{(\gamma(\widetilde{I-\mathbf{t}}))^{c}}\left(u^{\prime}\right) .
$$

This gives

$$
\begin{aligned}
\lambda_{n, I}(w)= & (l(u)+l(v))+l_{\mathrm{L}}^{(\gamma \widetilde{I})^{c}}(w) \\
= & 2 \sum_{\rho \in[r]} \gamma t_{\rho}\left(\gamma\left(i_{\rho+1}-i_{\rho}\right)-\gamma\left(t_{\rho+1}-t_{\rho}\right)\right) \\
& +\lambda_{\gamma(n-j), I-\mathbf{t}}\left(u^{\prime}\right)+\sum_{\rho \in[r+1]} l\left(v_{s}\right) .
\end{aligned}
$$

Conversely, any $\mathbf{t} \in T$ and any permutations $v_{1}, \ldots, v_{r+1}, u^{\prime}$ of the appropriate degrees such that (3.7) holds give rise to a permutation $w$ satisfying

$$
D_{\mathrm{L}}(w) \subseteq\left(D_{\mathrm{L}}\left(u^{\prime}\right)+\gamma j\right) \cup\{\gamma j\} .
$$

Thus (3.8) shows that the right-hand side of (3.5) is indeed equal to the right-hand side of (3.1).

\section{The ORTHOgOnAL CASE}

Our aim in this section is to complete the proof of Theorem A, We consider non-degenerate quadratic spaces $\mathcal{V}=(V, B, f)$, where $V$ is an $n$-dimensional vector space over the finite field $F=\mathbb{F}_{q}$, equipped with a quadratic form $f$, and $B$ denotes the bilinear form obtained by polarising $f$. So for all $x, y \in V$,

$$
B(x, y)= \begin{cases}f(x+y)+f(x)+f(y) & \text { if } \operatorname{char} F=2, \\ \frac{1}{2}(f(x+y)-f(x)-f(y)) & \text { if } \operatorname{char} F \neq 2 .\end{cases}
$$

If char $F \neq 2$, then $B$ is non-degenerate symmetric and, as $f(x)=B(x, x)$ for all $x \in V$, the quadratic form $f$ can easily be recovered from $B$. If char $F=2$, then $B$ is alternating, possibly degenerate and carries less information than $f$.

Non-degenerate quadratic spaces over finite fields have been classified and can be described up to isomorphism as follows; cf. 1, p. 144], [2, Section 3.3]. If 
char $F \neq 2$, then for any given dimension $n$ there are two possible isomorphism types of non-degenerate quadratic spaces $\mathcal{V}=(V, B, f)$, namely

$$
\begin{array}{cl}
\text { for } n \text { odd: } & \mathcal{V}=\mathcal{H}_{1} \perp \cdots \perp \mathcal{H}_{m} \perp \mathcal{A}_{1,1}, \\
\text { for } n \text { even: } & \mathcal{V}=\mathcal{H}_{1} \perp \cdots \perp \mathcal{H}_{m} \perp \mathcal{A}_{1,-1}, \\
& \mathcal{V}=\mathcal{H}_{1} \perp \cdots \perp \mathcal{H}_{m-1} \perp \mathcal{H}_{m}, \\
& \mathcal{V}=\mathcal{H}_{1} \perp \cdots \perp \mathcal{H}_{m-1} \perp \mathcal{A}_{2},
\end{array}
$$

where $m=\left\lfloor\frac{n}{2}\right\rfloor$, the $\mathcal{H}_{i}$ denote hyperbolic planes, $\mathcal{A}_{1,1}$ (respectively $\mathcal{A}_{1,-1}$ ) stands for an anisotropic line $\langle x\rangle$ with $f(x) \in\left(F^{*}\right)^{2}$ (respectively $f(x) \in F^{*} \backslash\left(F^{*}\right)^{2}$ ) and $\mathcal{A}_{2}$ is an anisotropic plane. For the purpose of counting non-degenerate flags in quadratic spaces $\mathcal{V}$ of given odd dimension, there is no significant difference between the two possible isomorphism types.

We now discuss the case char $F=2$. Then the above list still provides all isomorphism types of non-degenerate quadratic spaces, but becomes one term shorter: as every element of $F$ is a square, in any given odd dimension there is (up to isomorphism) just one non-degenerate quadratic space. In any given even dimension there are still two isomorphism types. Note also that non-degenerate quadratic spaces of odd dimension are defective with a 1-dimensional radical, whereas non-degenerate quadratic spaces of even dimension are non-defective.

Returning to the task of proving Theorem $\mathrm{A}$, we recall from the introduction that, in the even-dimensional case, we attach a sign $\varepsilon=1$ or $\varepsilon=-1$ to $\mathcal{V}$ according to whether the anisotropic kernel of $\mathcal{V}$ is 0 - or 2-dimensional. More suggestively, we write $a_{2 m+1}^{J}(q):=a_{\mathcal{V}}^{J}(q)$ if $n=2 m+1$ is odd and, similarly, $a_{2 m, \varepsilon}^{J}(q):=a_{\mathcal{V}}^{J}(q)$ if $n=2 m$ is even and $\mathcal{V}$ is of type $\varepsilon$. We are interested in the polynomials

$$
\begin{aligned}
\alpha_{2 m+1}^{J}\left(q^{-1}\right) & :=a_{2 m+1}^{J}(q) / q^{\operatorname{deg}_{q} a_{2 m+1}^{J}}, \\
\alpha_{2 m, \varepsilon}^{J}\left(q^{-1}\right) & :=a_{2 m, \varepsilon}^{J}(q) / q^{\operatorname{deg}_{q} a_{2 m, \varepsilon}^{J}} .
\end{aligned}
$$

Definition 4.1. Given a family $\mathbf{F}=\left(F_{J}(\mathbf{X})\right)_{J \subseteq[n-1]}$ of rational functions with the inversion property (IP) we define, respectively,

$$
\begin{aligned}
\operatorname{Ig}_{2 m+1}\left(q^{-1}, \mathbf{X}\right) & :=\operatorname{Ig}_{2 m+1, \mathbf{F}}\left(q^{-1}, \mathbf{X}\right):=\sum_{J \subseteq[n-1]} \alpha_{2 m+1}^{J}\left(q^{-1}\right) F_{J}(\mathbf{X}), \\
\operatorname{Ig}_{2 m, \varepsilon}\left(q^{-1}, \mathbf{X}\right) & :=\operatorname{Ig}_{2 m, \varepsilon, \mathbf{F}}\left(q^{-1}, \mathbf{X}\right):=\sum_{J \subseteq[n-1]} \alpha_{2 m, \varepsilon}^{J}\left(q^{-1}\right) F_{J}(\mathbf{X}) .
\end{aligned}
$$

To streamline notation, we will sometimes add in the odd-dimensional case a superfluous $\varepsilon$ to expressions such as $a_{n}^{J}(q), \alpha_{n}^{J}\left(q^{-1}\right)$ or $\operatorname{Ig}_{n}\left(q^{-1}, \mathbf{X}\right)$, thus writing e.g. $a_{n, \varepsilon}^{J}(q), \alpha_{n, \varepsilon}^{J}\left(q^{-1}\right)$ or $\operatorname{Ig}_{n, \varepsilon}\left(q^{-1}, \mathbf{X}\right)$, irrespective of the parity of $n$.

We now fix a family of rational functions $\mathbf{F}=\left(F_{J}(\mathbf{X})\right)_{J \subseteq[n-1]}$ with the inversion property (IP). The assertion of Theorem $\mathrm{A}$ in the orthogonal case then takes the following form.

Theorem 4.2. The Igusa-type functions satisfy functional equations

$$
\begin{aligned}
\operatorname{Ig}_{2 m+1}\left(q, \mathbf{X}^{-1}\right) & =(-1)^{m} q^{m^{2}+m} \operatorname{Ig}_{2 m+1}\left(q^{-1}, \mathbf{X}\right), \\
\operatorname{Ig}_{2 m, \varepsilon}\left(q, \mathbf{X}^{-1}\right) & =-\varepsilon(-1)^{m} q^{m^{2}} \operatorname{Ig}_{2 m, \varepsilon}\left(q^{-1}, \mathbf{X}\right) .
\end{aligned}
$$

We first give an outline of the proof of Theorem 4.2, deferring precise definitions for a moment. In Proposition 4.5 we derive explicit formulae for the polynomials 
$\alpha_{n, \varepsilon}^{J}\left(q^{-1}\right)$ from the well-known formulae for the orders of the orthogonal groups. A key observation is that the map $J \mapsto \alpha_{n, \varepsilon}^{J}\left(q^{-1}\right)$ factors over a 'bisecting' map $\varphi: \mathcal{P}([n]) \rightarrow \mathcal{P}([m])$. We are thus led to define, for $G \subseteq[m], I \in \varphi^{-1}(G)$,

$$
\alpha_{n, \varepsilon}^{\uparrow G}\left(q^{-1}\right):=\alpha_{n, \varepsilon}^{I}\left(q^{-1}\right)
$$

and

$$
F_{\varphi^{-1}(G)}(\mathbf{X}):=\sum_{I \in \varphi^{-1}(G)} F_{I}(\mathbf{X})
$$

so that

$$
\operatorname{Ig}_{n, \varepsilon}\left(q^{-1}, \mathbf{X}\right)=\sum_{G \subseteq[m]} \alpha_{n, \varepsilon}^{\uparrow G}\left(q^{-1}\right) F_{\varphi^{-1}(G)}(\mathbf{X}) .
$$

As we shall see, any subset $G \subseteq[m]$ induces in a natural way a composition $C(G):=$ $C(G, m)$ of a non-negative integer $N(G) \leq m$. For $G, H \subseteq[m]$, we denote by $\|G\|$ the number of parts of $C(G)$ and by $c_{G, H}$ the number of ways the composition $C(H)$ refines a truncation of the composition $C(G)$. We then prove the following 'inversion equations'.

Proposition 4.3. $\quad$ (i) For each $H \subseteq[m]$,

$$
F_{\varphi^{-1}(H)}\left(\mathbf{X}^{-1}\right)=(-1)^{n-1+\|H\|} \sum_{G \subseteq[m]} c_{G, H} F_{\varphi^{-1}(G)}(\mathbf{X}) .
$$

(ii) For each $G \subseteq[m]$,

$$
\begin{aligned}
\alpha_{2 m+1}^{\uparrow G}(q) & =(-1)^{m} q^{m^{2}+m} \sum_{H \subseteq[m]}(-1)^{\|H\|} c_{G, H} \alpha_{2 m+1}^{\uparrow H}\left(q^{-1}\right), \\
\alpha_{2 m, \varepsilon}^{\uparrow G}(q) & =\varepsilon(-1)^{m} q^{m^{2}} \sum_{H \subseteq[m]}(-1)^{\|H\|} c_{G, H} \alpha_{2 m, \varepsilon}^{\uparrow H}\left(q^{-1}\right) .
\end{aligned}
$$

Theorem 4.2 is an immediate consequence of Proposition 4.3 indeed, in the odd-dimensional case,

$$
\begin{aligned}
\operatorname{Ig}_{2 m+1}\left(q, \mathbf{X}^{-1}\right) & =\sum_{G \subseteq[m]} \alpha_{2 m+1}^{\uparrow G}(q) F_{\varphi^{-1}(G)}\left(\mathbf{X}^{-1}\right) \\
& =(-1)^{m} q^{m^{2}+m} \sum_{G, H \subseteq[m]}(-1)^{\|H\|} c_{G, H} \alpha_{2 m+1}^{\uparrow H}\left(q^{-1}\right) F_{\varphi^{-1}(G)}\left(\mathbf{X}^{-1}\right) \\
& =(-1)^{m} q^{m^{2}+m} \sum_{H \subseteq[m]} \alpha_{2 m+1}^{\uparrow H}\left(q^{-1}\right) F_{\varphi^{-1}(H)}(\mathbf{X}) \\
& =(-1)^{m} q^{m^{2}+m} \operatorname{Ig}_{2 m+1}\left(q^{-1}, \mathbf{X}\right)
\end{aligned}
$$

The functional equation for $\operatorname{Ig}_{2 m, \varepsilon}\left(q^{-1}, \mathbf{X}\right)$ follows in a similar way. In the remainder of this section we give precise definitions of the above concepts, and we supply a proof of Proposition 4.3 .

Definition 4.4 (Integer compositions). By a composition $C$ of a non-negative integer $N$ into $\rho$ parts we mean a tuple $\left(x_{1}, \ldots, x_{\rho}\right) \in \mathbb{N}^{\rho}$ such that $N=x_{1}+\cdots+x_{\rho}$.

Given $I=\left\{i_{1}, \ldots, i_{r}\right\}_{<} \subseteq[n]$, we define

$$
N(I, n):=\max \left([n]_{0} \backslash I\right) \text { and } \rho:=\max \left\{\varrho \in[r+1]_{0} \mid i_{\varrho-1}<N(I, n)\right\},
$$


where $i_{-1}:=-1, i_{0}:=0$. Then $I$ induces a composition $C(I, n)$ of $N(I, n)$ into $\|I\|_{n}:=\rho$ parts; namely,

$$
C(I, n):=\left(i_{1}, i_{2}-i_{1}, \ldots, i_{\rho-1}-i_{\rho-2}, N(I, n)-i_{\rho-1}\right) .
$$

Note that, if $I \subseteq[n-1]$, then $N(I, n)=n$ and $\rho=r+1$. The map $I \mapsto C(I, n)$ induces a bijection from $\mathcal{P}([n-1])$ onto the set of all compositions of $n$.

We define the bisecting map

$$
\varphi: \mathcal{P}([n]) \rightarrow \mathcal{P}([m])
$$

as follows: for $I \subseteq[n]$ with $C(I, n)=\left(x_{1}, \ldots, x_{\rho}\right)$ set

$$
\begin{aligned}
\operatorname{cut}(I) & :=\left\lfloor\frac{x_{1}}{2}\right\rfloor+\left\lfloor\frac{x_{2}}{2}\right\rfloor+\cdots+\left\lfloor\frac{x_{\rho}}{2}\right\rfloor, \\
\varphi_{0}(I) & :=\left\{\left\lfloor\frac{x_{1}}{2}\right\rfloor+\left\lfloor\frac{x_{2}}{2}\right\rfloor+\cdots+\left\lfloor\frac{x_{\varrho}}{2}\right\rfloor \mid \varrho \in[\rho]\right\} \backslash\{0, \operatorname{cut}(I)\}
\end{aligned}
$$

and

$$
\varphi(I):=\varphi_{0}(I) \cup[\operatorname{cut}(I)+1, m] .
$$

Note that $N(\varphi(I), m)=\operatorname{cut}(I)$ and $\|\varphi(I)\|_{m} \leq\|I\|_{n}$. Moreover, $\varphi$ maps $\mathcal{P}([n-1])$ surjectively onto $\mathcal{P}([m])$. For subsets $G \subseteq[m]$ we agree to write $N(G):=N(G, m)$ and $\|G\|:=\|G\|_{m}$.

We now give explicit formulae for the polynomials $\alpha_{n, \varepsilon}^{J}\left(q^{-1}\right)$.

Proposition 4.5. Let $J \subseteq[n-1], H:=\varphi(J) \subseteq[m]$, and put $Y:=q^{-2}$.

(i) For $n=2 m+1$ odd,

$$
\begin{aligned}
\alpha_{2 m+1}^{J}\left(q^{-1}\right) & =\left(\begin{array}{l}
N(H) \\
\varphi_{0}(J)
\end{array}\right)_{Y} \prod_{i=N(H)+1}^{m}\left(1-Y^{i}\right) \\
& =\left(\begin{array}{c}
m \\
H \cup\{N(H)\}
\end{array}\right)_{Y}(1-Y)^{m-N(H)} .
\end{aligned}
$$

(ii) For $n=2 m$ even,

$$
\begin{array}{rlrl}
\alpha_{2 m, \varepsilon}^{J}\left(q^{-1}\right) & =\left(\begin{array}{c}
m \\
J / 2
\end{array}\right)_{Y}=\left(\begin{array}{l}
m \\
H
\end{array}\right)_{Y} & & \text { if } J \subseteq 2 \mathbb{N}, \\
\alpha_{2 m, \varepsilon}^{J}\left(q^{-1}\right) & =\left(\begin{array}{c}
N(H) \\
\varphi_{0}(J)
\end{array}\right)_{Y} \frac{\prod_{i=N(H)+1}^{m}\left(1-Y^{i}\right)}{1+\varepsilon q^{-m}} & \\
& =\left(\begin{array}{c}
m \\
H \cup\{N(H)\}
\end{array}\right)_{Y} \frac{(1-Y)^{m-N(H)}}{1+\varepsilon q^{-m}} & \text { otherwise. }
\end{array}
$$

Proof. First we are going to prove the assertions in odd characteristic, where the discriminant helps to distinguish isomorphism types of quadratic spaces and where we can freely apply Witt's Extension and Cancellation Theorem. Afterwards we explain why the formulae also remain true in characteristic 2 .

So first suppose that the underlying field $F=\mathbb{F}_{q}$ has odd characteristic. Recall the formulae for the orders of the respective orthogonal groups:

$$
\begin{aligned}
\left|\mathrm{O}_{2 m+1}\left(\mathbb{F}_{q}\right)\right| & =2 q^{m^{2}} \prod_{i \in[m]}\left(q^{2 i}-1\right)=: p_{2 m+1}(q)=: p_{2 m+1}, \\
\left|\mathrm{O}_{2 m}^{\varepsilon}\left(\mathbb{F}_{q}\right)\right| & =2 q^{m^{2}-m}\left(q^{m}-\varepsilon\right) \prod_{i \in[m-1]}\left(q^{2 i}-1\right)=: p_{2 m, \varepsilon}(q)=: p_{2 m, \varepsilon}
\end{aligned}
$$


(cf. [1, p. 147], [9, Theorem 9.11]), and put

$$
p_{n}^{\sharp}:=p_{n}^{\sharp}(q):= \begin{cases}p_{2 m+1} & \text { for } n=2 m+1 \text { odd, } \\ \left(q^{m}+\varepsilon\right) p_{2 m, \varepsilon} & \text { for } n=2 m \text { even. }\end{cases}
$$

Let $J=\left\{j_{1}, \ldots, j_{s}\right\}_{<} \subseteq[n-1]$, and put $j_{0}:=0, j_{s+1}:=n$. Counting nondegenerate flags $\mathbf{U}_{J}=\left(U_{j}\right)_{j \in J}$ of type $J$ in $\mathcal{V}$ is equivalent to counting (ordered) orthogonal decompositions

$$
\mathcal{V}=\mathcal{W}_{1} \perp \cdots \perp \mathcal{W}_{s+1}
$$

with $\operatorname{dim} \mathcal{W}_{\sigma}=k_{\sigma}:=j_{\sigma}-j_{\sigma-1}$ for all $\sigma \in[s+1]$. The isomorphism type of such an orthogonal decomposition is determined by the discriminants $\operatorname{disc} \mathcal{W}_{\sigma} \in$ $\mathbb{F}_{q}^{*} /\left(\mathbb{F}_{q}^{*}\right)^{2} \cong\{1,-1\}$ of the non-degenerate spaces $\mathcal{W}_{\sigma}, \sigma \in[s+1]$.

Let $\eta \in\{1,-1\}$, according to whether -1 is a square in $\mathbb{F}_{q}$ or not. At this point it is advantageous to assign, also to an odd-dimensional non-degenerate quadratic space $\mathcal{W}$ a $\operatorname{sign} \varepsilon(\mathcal{W}) \in\{1,-1\}$, namely the discriminant of the (one-dimensional) anisotropic kernel of $\mathcal{W}$. We then have $\operatorname{disc} \mathcal{W}=\varepsilon(\mathcal{W}) \eta^{\lfloor\operatorname{dim} \mathcal{W} / 2\rfloor}$ for any nondegenerate quadratic space $\mathcal{W}$, irrespective of the parity of $\operatorname{dim} \mathcal{W}$.

Thus the isomorphism type of an orthogonal decomposition of the form (4.1) can be encoded in a tuple $\varepsilon=\left(\varepsilon_{1}, \ldots, \varepsilon_{s+1}\right) \in\{1,-1\}^{s+1}$ such that $\mathcal{W}_{\sigma}$ is of type $\varepsilon_{\sigma}$ for all $\sigma \in[s+1]$. Moreover, the tuples $\varepsilon$ which arise in this way are precisely the elements of $E:=E(\mathcal{V}):=\left\{\varepsilon \mid \varepsilon_{1} \cdots \varepsilon_{s+1}=\eta^{m-N(\varphi(J))} \varepsilon\right\}$, and Witt's Extension and Cancellation Theorem implies that the number of ordered orthogonal decompositions of isomorphism type $\varepsilon \in E$ equals

$$
\frac{\left|\mathrm{O}_{n}^{\varepsilon}\left(\mathbb{F}_{q}\right)\right|}{\left|\prod_{\sigma=1}^{s+1} \mathrm{O}_{k_{\sigma}}^{\varepsilon_{\sigma}}\left(\mathbb{F}_{q}\right)\right|}=\frac{p_{n, \varepsilon}}{\prod_{\sigma=1}^{s+1} p_{k_{\sigma}, \varepsilon_{\sigma}}} ;
$$

cf. [1, p. 147f]. Setting

$$
\mathcal{E}(J):=\left\{\sigma \in[s+1] \mid k_{\sigma} \equiv 0 \bmod 2\right\}
$$

we thus obtain

$$
a_{n, \varepsilon}^{J}(q)=p_{n, \varepsilon} \sum_{\varepsilon \in E}\left(\prod_{\sigma=1}^{s+1} p_{k_{\sigma}, \varepsilon_{\sigma}}\right)^{-1}=\frac{p_{n, \varepsilon}}{\prod_{\sigma=1}^{s+1} p_{k_{\sigma}}^{\sharp}} \sum_{\boldsymbol{\varepsilon} \in E} \prod_{\sigma \in \mathcal{E}(J)}\left(q^{k_{\sigma} / 2}+\varepsilon_{\sigma}\right) .
$$

Note that

$$
\begin{array}{ll}
\sum_{\varepsilon \in E} \prod_{\sigma \in \mathcal{E}(J)}\left(q^{k_{\sigma} / 2}+\varepsilon_{\sigma}\right)=2^{s} \prod_{\sigma \in \mathcal{E}(J)} q^{k_{\sigma} / 2} & \text { if } \mathcal{E}(J) \neq[s+1], \\
\sum_{\boldsymbol{\varepsilon} \in E} \prod_{\sigma \in \mathcal{E}(J)}\left(q^{k_{\sigma} / 2}+\varepsilon_{\sigma}\right)=2^{s}\left(q^{n / 2}+\varepsilon\right) & \text { if } \mathcal{E}(J)=[s+1] .
\end{array}
$$

From this the claim follows for char $F \neq 2$.

Before turning our attention to the case $\operatorname{char} F=2$, we record a set of formulae for later use. Let $j \in[n-1]$ and $\delta \in\{1,-1\}$. If $j=2 h+1$ is odd, let $a_{\mathcal{V}}^{j}(q)$ denote the number of non-degenerate $j$-dimensional subspaces in $\mathcal{V}$. If $j=2 h$ is even, let $a_{\mathcal{V}}^{j, \delta}(q)$ denote the number of non-degenerate $j$-dimensional subspaces of type $\delta$ in $\mathcal{V}$. According to whether $\mathcal{V}$ is odd- or even-dimensional, we also write $a_{2 m+1}^{2 h+1}(q)$, $a_{2 m, \varepsilon}^{2 h+1}(q)$ in the former and $a_{2 m+1}^{2 h, \delta}(q), a_{2 m, \varepsilon}^{2 h, \delta}(q)$ in the latter case. Our calculations 
above, based on Witt's Extension and Cancellation Theorem, show in particular that, if $\operatorname{char} F \neq 2$,

$$
\begin{aligned}
a_{2 m+1}^{2 h+1}(q) & =\frac{\left|\mathrm{O}_{2 m+1}\left(\mathbb{F}_{q}\right)\right|}{\left|\mathrm{O}_{2 h+1}\left(\mathbb{F}_{q}\right)\right|\left|\mathrm{O}_{2 m-2 h}^{+}\left(\mathbb{F}_{q}\right)\right|}+\frac{\left|\mathrm{O}_{2 m+1}\left(\mathbb{F}_{q}\right)\right|}{\left|\mathrm{O}_{2 h+1}\left(\mathbb{F}_{q}\right)\right|\left|\mathrm{O}_{2 m-2 h}^{-}\left(\mathbb{F}_{q}\right)\right|} \\
& =\frac{2 q^{m-h} p_{2 m+1}}{p_{2 h+1} p_{2 m-2 h}^{\sharp}}, \\
a_{2 m, \varepsilon}^{2 h+1}(q) & =2 \cdot \frac{\left|\mathrm{O}_{2 m}^{\varepsilon}\left(\mathbb{F}_{q}\right)\right|}{\left|\mathrm{O}_{2 h+1}\left(\mathbb{F}_{q}\right)\right|\left|\mathrm{O}_{2 m-2 h-1}\left(\mathbb{F}_{q}\right)\right|}=\frac{2 p_{2 m, \varepsilon}}{p_{2 h+1} p_{2 m-2 h-1}}, \\
a_{2 m+1}^{2 h, \delta}(q) & =\frac{\left|\mathrm{O}_{2 m+1}\left(\mathbb{F}_{q}\right)\right|}{\left|\mathrm{O}_{2 h}^{\delta}\left(\mathbb{F}_{q}\right)\right|\left|\mathrm{O}_{2 m-2 h+1}\left(\mathbb{F}_{q}\right)\right|}=\frac{p_{2 m+1}}{p_{2 h, \delta} p_{2 m-2 h+1}}, \\
a_{2 m, \varepsilon}^{2 h, \delta}(q) & =\frac{\left|\mathrm{O}_{2 m}^{\varepsilon}\left(\mathbb{F}_{q}\right)\right|}{\left|\mathrm{O}_{2 h}^{\delta}\left(\mathbb{F}_{q}\right)\right|\left|\mathrm{O}_{2 m-2 h}^{\delta \varepsilon}\left(\mathbb{F}_{q}\right)\right|}=\frac{p_{2 m, \varepsilon}}{p_{2 h, \delta} p_{2 m-2 h, \delta \varepsilon}} .
\end{aligned}
$$

Below we will show that, in fact, also in characteristic 2 one obtains the same polynomials $a_{\mathcal{V}}^{j}(q)$ and $a_{\mathcal{V}}^{j, \delta}(q)$. Thus, by induction, the formulae for $a_{\mathcal{V}}^{J}(q)$ and $\alpha_{\mathcal{V}}^{J}\left(q^{-1}\right)$, which we initially derived only under the extra assumption $\operatorname{char} F \neq 2$, also remain valid in characteristic 2 .

So suppose that char $F=2$, and let $j \in[n-1], \delta \in\{1,-1\}$. Write $j=2 h+1$ if $j$ is odd, and $j=2 h$ if $j$ is even. The orders of the respective orthogonal groups are now

$$
\left|\mathrm{O}_{2 m+1}\left(\mathbb{F}_{q}\right)\right|=\frac{p_{2 m+1}}{2}, \quad\left|\mathrm{O}_{2 m}^{\varepsilon}\left(\mathbb{F}_{q}\right)\right|=p_{2 m, \varepsilon},
$$

and Witt's Extension and Cancellation Theorem still applies to non-defective subspaces; cf. [9, Theorems 3.12 and 14.48] and [2, Theorem 3.15]. Therefore we immediately obtain the counterparts of (4.4) and (4.5),

$$
\begin{aligned}
a_{2 m+1}^{2 h, \delta}(q) & =\frac{\left|\mathrm{O}_{2 m+1}\left(\mathbb{F}_{q}\right)\right|}{\left|\mathrm{O}_{2 h}^{\delta}\left(\mathbb{F}_{q}\right)\right|\left|\mathrm{O}_{2 m-2 h+1}\left(\mathbb{F}_{q}\right)\right|}=\frac{p_{2 m+1}}{p_{2 h, \delta} p_{2 m-2 h+1}}, \\
a_{2 m, \varepsilon}^{2 h, \delta}(q) & =\frac{\left|\mathrm{O}_{2 m}^{\varepsilon}\left(\mathbb{F}_{q}\right)\right|}{\left|\mathrm{O}_{2 h}^{\delta}\left(\mathbb{F}_{q}\right)\right|\left|\mathrm{O}_{2 m-2 h}^{\delta \varepsilon}\left(\mathbb{F}_{q}\right)\right|}=\frac{p_{2 m, \varepsilon}}{p_{2 h, \delta} p_{2 m-2 h, \delta \varepsilon}} .
\end{aligned}
$$

Next we suppose that $n=2 m+1$ is odd and compute $a_{2 m+1}^{2 h+1}(q)$. If $h=0$, then we are to count anisotropic lines in $\mathcal{V}$. It is well known that the polar space associated to $\mathcal{V}$ has $\left(q^{2 m}-1\right) /(q-1)$ points, each corresponding to an isotropic line; cf. 2, Theorem 3.13]. So we deduce that

$$
a_{2 m+1}^{1}(q)=\frac{q^{2 m+1}-1}{q-1}-\frac{q^{2 m}-1}{q-1}=\frac{2 q^{m} p_{2 m+1}}{p_{1} p_{2 m}^{\sharp}} .
$$

In general, choosing a $(2 h+1)$-dimensional non-degenerate subspace $U$ in $\mathcal{V}$ can be split into two parts: first pick a $2 h$-dimensional non-degenerate (hence nondefective) subspace $U_{0}$ of type 1 , then complement your choice by picking an anisotropic line $A$ in $U_{0}^{\perp}$ to obtain $U=U_{0}+A$. Applying Witt's Extension and 
Cancellation Theorem, we obtain the counterpart of (4.2),

$$
\begin{aligned}
a_{2 m+1}^{2 h+1}(q) & =\frac{a_{2 m+1}^{2 h, 1}(q) a_{2 m-2 h+1}^{1}(q)}{a_{2 h+1}^{2 h, 1}(q)} \\
& =\frac{\left(p_{2 m+1} \cdot 2 q^{m-h} p_{2 m-2 h+1}\right) /\left(p_{2 h, 1} p_{2 m-2 h+1} \cdot p_{1} p_{2 m-2 h}^{\sharp}\right)}{\left(p_{2 h+1}\right) /\left(p_{2 h, 1} p_{1}\right)} \\
& =\frac{2 q^{m-h} p_{2 m+1}}{p_{2 h+1} p_{2 m-2 h}^{\sharp}} .
\end{aligned}
$$

A similar computation yields the counterpart of (4.3).

Definition 4.6 (Refinements of compositions). Let $C_{1}=\left(x_{1}, \ldots, x_{\kappa}\right)$ and $C_{2}=$ $\left(y_{1}, \ldots, y_{\lambda}\right)$ be compositions. A refinement of a truncation of $C_{1}$ by $C_{2}$ is a triple $\left(C_{1}, C_{2}, \boldsymbol{\xi}\right)$ such that $\boldsymbol{\xi}=\left(\xi_{1}, \ldots, \xi_{\kappa}\right) \in[\lambda]_{0}{ }^{\kappa}$ satisfies

$$
\xi_{1} \leq \cdots \leq \xi_{\kappa}=\lambda \text { and } \forall i \in[\kappa]: y_{\xi_{i-1}+1}+\cdots+y_{\xi_{i}} \leq x_{i},
$$

where $\xi_{0}:=0$. By a slight abuse of terminology, we also call the $\kappa$-tuple $\boldsymbol{\xi}$ a refinement of a truncation of $C_{1}$ by $C_{2}$. For $G, H \subseteq[m]$, the number of refinements of truncations of $C(G)$ by $C(H)$ is denoted by $c_{G, H}:=c_{G, H}^{(m)}$.

Let $I, J \subseteq[n-1]$ such that $I \subseteq J$, and put $G:=\varphi(I), H:=\varphi(J)$. Clearly, $C(J, n)$ can be regarded as a refinement of $C(I, n)$. Applying the bisecting map, we obtain a refinement of a truncation of $C(G)$ by $C(H)$ as follows.

The sets $[n-1] \backslash I$ and $[n-1] \backslash J$ decompose uniquely into disjoint unions

$$
[n-1] \backslash I=\mathcal{I}_{I, 1} \dot{\cup} \ldots \dot{\cup} \mathcal{I}_{I,\|G\|}, \quad[n-1] \backslash J=\mathcal{I}_{J, 1} \dot{\cup} \ldots \dot{\cup} \mathcal{I}_{J,\|H\|}
$$

of intervals $\mathcal{I}_{I, i}$ (respectively $\mathcal{I}_{J, j}$ ) of natural numbers with $\max \mathcal{I}_{I, i}<\min \mathcal{I}_{I, i+1}$ (respectively $\max \mathcal{I}_{J, j}<\min \mathcal{I}_{J, j+1}$ ) for all admissible values of $i$ (respectively $j$ ).

The refinement of a truncation of $G$ by $H$ induced from $I \subseteq J$ is the $\|G\|$-tuple $\boldsymbol{\xi}(I, J)=\left(\xi_{1}, \ldots, \xi_{\|G\|}\right)$ defined by $\xi_{\|G\|}:=\|H\|$ and

$$
\forall i \in[\|G\|]: \mathcal{I}_{J, \xi_{i-1}+1} \dot{\cup} \ldots \dot{\cup} \mathcal{I}_{J, \xi_{i}} \subseteq \mathcal{I}_{I, i},
$$

where $\xi_{0}:=0$. We remark that, starting from $G, H \subseteq[m]$, every refinement of a truncation of $C(G)$ by $C(H)$ is induced by suitable $I, J \subseteq[n-1]$ with $I \subseteq J$.

We illustrate these notions by an example.

Example 4.7. Set $n=11$ so that $m=5$. The subsets $G=\{1,3,4\}, H=$ $\{2,4,5\} \subseteq[m]$ induce compositions $C(G)=(1,2,1,1)$ of $N(G)=5$ and $C(H)=$ $(2,1)$ of $N(H)=3$, respectively. Note that $\|G\|=4$ and $\|H\|=2$. Among the seven 'truncations' of $(1,2,1,1)$ to 'pre-compositions' of 3 ,

$$
\begin{array}{llll}
(1,2,0,0), & (1,1,1,0), \quad(1,1,0,1), \quad(1,0,1,1), \quad(0,1,1,1), \\
(0,2,0,1), & (0,2,1,0),
\end{array}
$$

only the $2=c_{G, H}$ last ones yield the composition $C(H)=(2,1)$. They are encoded in the tuples $\boldsymbol{\xi}=(0,1,1,2)$ and $\boldsymbol{\xi}=(0,1,2,2)$, respectively.

Define subsets

$$
I:=\{2,7,9\}, \quad J_{1}:=\{1,2,7,8,9\}, \quad J_{2}:=\{1,2,3,7,9,10\}
$$

of $[n-1]=[10]$. The set $I$ induces the composition $C(I, n)=(2,5,2,2)$ of $N(I, n)=$ 11. Thus $\operatorname{cut}(I)=1+2+1+1=5$ and $\varphi(I)=G$. Similarly, $\operatorname{cut}\left(J_{1}\right)=\operatorname{cut}\left(J_{2}\right)=$ 
$2+1=3$ and $\varphi\left(J_{1}\right)=\varphi\left(J_{2}\right)=H$. We have $\boldsymbol{\xi}\left(I, J_{1}\right)=(0,1,1,2)$ and $\boldsymbol{\xi}\left(I, J_{2}\right)=$ $(0,1,2,2)$.

We are now ready to prove Proposition 4.3 ,

Proof of Proposition 4.3 (i). Let $H \subseteq[m]$. From the definition of $F_{\varphi^{-1}(H)}(\mathbf{X})$ and the fact that $\mathbf{F}$ has the inversion property (IP) we obtain

$$
F_{\varphi^{-1}(H)}\left(\mathbf{X}^{-1}\right)=\sum_{J \in \varphi^{-1}(H)}(-1)^{|J|} \sum_{I \subseteq J} F_{I}(\mathbf{X}) .
$$

Thus it is enough to show that for $I \subseteq[n-1]$ with $\varphi(I)=G$,

$$
(-1)^{n-1+\|H\|} \sum_{\substack{J \in \varphi^{-1}(H) \\ I \subseteq J}}(-1)^{|J|}=c_{G, H} .
$$

This is certainly the case if $c_{G, H}=0$, as then the sum on the left-hand side is empty. Now suppose that $c_{G, H} \neq 0$ and fix a refinement $\boldsymbol{\xi}$ of a truncation of $G$ by $H$; put $\xi_{0}:=0$. It suffices to show that

$$
(-1)^{n-1+\|H\|} \sum_{\substack{J \in \varphi^{-1}(H) \\ I \subseteq J, \boldsymbol{\xi}(I, J)=\boldsymbol{\xi}}}(-1)^{|J|}=1 .
$$

Decompose $[n-1] \backslash I=\mathcal{I}_{I, 1} \dot{\cup} \ldots \dot{\cup} \mathcal{I}_{I,\|G\|}$ into a disjoint union of intervals $\mathcal{I}_{I, i}$ as in Definition 4.6, and write $C(H)=\left(y_{1}, \ldots, y_{\|H\|}\right)$. We claim that

$$
\begin{array}{r}
\sum_{\substack{J \in \varphi^{-1}(H) \\
I \subseteq J, \boldsymbol{\xi}(I, J)=\boldsymbol{\xi}}}(-1)^{|J|-|I|}=\prod_{i=1}^{\|G\|} \sum_{k=0}^{\xi_{i}-\xi_{i-1}}\left(\begin{array}{c}
\left|\mathcal{I}_{I, i}\right|-\sum_{j=\xi_{i-1}+1}^{\xi_{i}}\left(2 y_{j}-1\right)-k+1 \\
\xi_{i}-\xi_{i-1}
\end{array}\right) \\
\cdot\left(\begin{array}{c}
\xi_{i}-\xi_{i-1} \\
k
\end{array}\right)(-1)^{\left|\mathcal{I}_{I, i}\right|-\sum_{j=\xi_{i-1}+1}^{\xi_{i}}\left(2 y_{j}-1\right)-k} .
\end{array}
$$

Indeed, specifying $J \in \varphi^{-1}(H)$ with $I \subseteq J$ and $\boldsymbol{\xi}(I, J)=\boldsymbol{\xi}$ is equivalent to the following task: for each $i \in[\|G\|]$ choose $k_{i} \in\left[\xi_{i}-\xi_{i-1}\right]_{0}$ and single out a disjoint union $\mathcal{I}_{J, \xi_{i-1}+1} \dot{\cup} \ldots \dot{\cup} \mathcal{I}_{J, \xi_{i}} \subseteq \mathcal{I}_{I, i}$ of intervals $\mathcal{I}_{J, j}$ such that

(a) $\max \mathcal{I}_{J, j}<\min \mathcal{I}_{J, j+1}$ for all admissible values of $j$,

(b) $\left|\mathcal{I}_{J, j}\right|=2 y_{j}$ for exactly $k_{i}$ values of $j$ and $\left|\mathcal{I}_{J, j}\right|=2 y_{j}-1$ for the remaining values of $j$.

Moreover, the cardinality of the set $J$ corresponding to such a choice of $k_{i}$ and such a choice of intervals $\mathcal{I}_{J, j} \subseteq \mathcal{I}_{I, i}$ is

$$
|I|+\sum_{i=1}^{\|G\|}\left(\left|\mathcal{I}_{I, i}\right|-\sum_{j=\xi_{i-1}+1}^{\xi_{i}}\left(2 y_{j}-1\right)-k_{i}\right) .
$$

As $n-1+\|H\|=|I|+\sum_{i=1}^{\|G\|}\left|\mathcal{I}_{I, i}\right|+\sum_{i=1}^{\|G\|}\left(\xi_{i}-\xi_{i-1}\right)$, equation (4.7) implies that the left-hand side of (4.6) is equal to

$$
\prod_{i=1}^{\|G\|} \sum_{k=0}^{\xi_{i}-\xi_{i-1}}\left(\begin{array}{c}
\left|\mathcal{I}_{I, i}\right|-\sum_{j=\xi_{i-1}+1}^{\xi_{i}}\left(2 y_{j}-1\right)+1-k \\
\xi_{i}-\xi_{i-1}
\end{array}\right)\left(\begin{array}{c}
\xi_{i}-\xi_{i-1} \\
k
\end{array}\right)(-1)^{k} .
$$


This does indeed equal 1, because for any positive integers $M \leq N$,

$$
\sum_{k=0}^{M}\left(\begin{array}{c}
N-k \\
M
\end{array}\right)\left(\begin{array}{c}
M \\
k
\end{array}\right)(-1)^{k}=1
$$

(cf. [8, p. 169, (5.25)]) and hence each of the $\|G\|$ factors already equals 1 .

Proof of Proposition 4.3 (ii) for $n=2 m+1$ odd. For $G \subseteq[m]$, we are looking to prove

$$
\alpha_{2 m+1}^{\uparrow G}(q)=(-1)^{m} q^{m^{2}+m} \sum_{H \subseteq[m]}(-1)^{\|H\|} c_{G, H} \alpha_{2 m+1}^{\uparrow H}\left(q^{-1}\right) .
$$

First we deal with the case $N(G)<m$, i.e. $m \in G$. Writing $G^{\prime}:=G \backslash\{m\}$ and $Y:=q^{-2}$, we see from Proposition 4.5 (i) that in this case

$$
\alpha_{2 m+1}^{\uparrow G}\left(q^{-1}\right)=\left(1-Y^{m}\right) \alpha_{2 m-1}^{\uparrow G^{\prime}}\left(q^{-1}\right) .
$$

If $H \subseteq[m]$ with $c_{G, H} \neq 0$, then $N(H) \leq N(G)<m$; hence $m \in H$, and hence we obtain $\|H\|=\left\|H^{\prime}\right\|_{m-1}$ and $c_{G, H}=c_{G^{\prime}, H^{\prime}}^{(m-1)}$ for $H^{\prime}:=H \backslash\{m\}$. With these observations (4.8) follows by induction:

$$
\begin{aligned}
\alpha_{2 m+1}^{\uparrow G}(q)= & \left(1-Y^{-m}\right)(-1)^{m-1} q^{(m-1)^{2}+(m-1)} \\
& \cdot \sum_{H^{\prime} \subseteq[m-1]}(-1)^{\left\|H^{\prime}\right\|_{m-1}} c_{G^{\prime}, H^{\prime}}^{(m-1)} \alpha_{2 m-1}^{\uparrow H^{\prime}}\left(q^{-1}\right) \\
= & (-1)^{m} q^{m^{2}+m} \sum_{H^{\prime} \subseteq[m-1]}(-1)^{\left\|H^{\prime}\right\|_{m-1}} c_{G^{\prime}, H^{\prime}}^{(m-1)}\left(1-Y^{m}\right) \alpha_{2 m-1}^{\uparrow H^{\prime}}\left(q^{-1}\right) \\
= & (-1)^{m} q^{m^{2}+m} \sum_{H \subseteq[m]}(-1)^{\|H\|} c_{G, H} \alpha_{2 m-1}^{\uparrow H}\left(q^{-1}\right) .
\end{aligned}
$$

It remains to consider the case $N(G)=m$, i.e. $G \subseteq[m-1]$. Again set $Y:=q^{-2}$, and write $C(G)=\left(x_{1}, \ldots, x_{k+1}\right)$. Proposition 4.5 (i) shows that in this case

$$
\alpha_{2 m+1}^{\uparrow G}\left(q^{-1}\right)=\left(\begin{array}{c}
m \\
G
\end{array}\right)_{Y}
$$

in particular, as $\operatorname{deg}_{Y}\left(\begin{array}{c}m \\ G\end{array}\right)_{Y}=\left(\begin{array}{c}m+1 \\ 2\end{array}\right)-\sum_{\kappa \in[k+1]}\left(\begin{array}{c}x_{\kappa}+1 \\ 2\end{array}\right)$,

$$
\alpha_{2 m+1}^{\uparrow G}(q)=\alpha_{2 m+1}^{\uparrow G}\left(q^{-1}\right) Y^{-\left(\begin{array}{c}
m+1 \\
2
\end{array}\right)+\sum_{\kappa \in[k+1]}\left({ }_{2}^{x_{\kappa}+1}\right)} .
$$

We shall show below that

$$
\alpha_{2 m+1}^{\uparrow G}\left(q^{-1}\right) Y^{\sum_{\kappa \in[k+1]}\left(\begin{array}{c}
x_{\kappa}+1 \\
2
\end{array}\right)}=\sum_{H \subseteq[m]}(-1)^{m+\|H\|} c_{G, H} \alpha_{2 m+1}^{\uparrow H}\left(q^{-1}\right) .
$$

From these equations (4.8) follows readily. 
It remains to prove (4.9). For this we need the following formulae.

(i) For all $i \in \mathbb{N}_{0}: Y^{\left(\begin{array}{c}i+1 \\ 2\end{array}\right)}=\sum_{j=0}^{i}\left(\begin{array}{c}i \\ {[i-j, i-1]}\end{array}\right) Y(Y-1)^{j} Y^{\left(\begin{array}{c}i-j \\ 2\end{array}\right)}$.

(ii) For all $i \in \mathbb{N}_{0}: Y^{\left(\begin{array}{c}i+1 \\ 2\end{array}\right)}=\sum_{I \subseteq[i]}\left(\begin{array}{c}i+1 \\ I\end{array}\right)_{Y}(-1)^{i-|I|}$.

Part (i) is easily proved inductively (see the end of this proof); part (ii) is a wellknown fact about Gaussian polynomials. With the formulae (i), (ii) at our disposal, the left-hand side of (4.9) can be written as

$$
\begin{aligned}
& \alpha_{2 m+1}^{\uparrow G}\left(q^{-1}\right) Y^{\sum_{\kappa \in[k+1]}\left(\begin{array}{c}
x_{\kappa}+1 \\
2
\end{array}\right)} \\
& \left.=\left(\begin{array}{c}
m \\
G
\end{array}\right)_{Y} \prod_{\kappa \in[k+1]} Y^{\left(x_{\kappa}+1\right.}\right) \\
& =\left(\begin{array}{c}
m \\
G
\end{array}\right)_{Y} \prod_{\kappa \in[k+1]}\left(\sum_{j=0}^{x_{\kappa}}\left(\begin{array}{c}
x_{\kappa} \\
{\left[x_{\kappa}-j, x_{\kappa}-1\right]}
\end{array}\right)_{Y}(Y-1)^{j} Y^{\left(x_{\kappa}-j\right)}\right) \\
& =\left(\begin{array}{c}
m \\
G
\end{array}\right)_{Y} \prod_{\kappa \in[k+1]}\left(\sum_{j=0}^{x_{\kappa}}\left(\begin{array}{c}
x_{\kappa} \\
{\left[x_{\kappa}-j, x_{\kappa}-1\right]}
\end{array}\right)_{Y}(1-Y)^{j}\right. \\
& \left.\sum_{K \subseteq\left[x_{\kappa}-j-1\right]}\left(\begin{array}{c}
x_{\kappa}-j \\
K
\end{array}\right)_{Y}(-1)^{x_{\kappa}+|K|+\delta\left({ }^{\prime} j \neq x_{\kappa} '\right)}\right),
\end{aligned}
$$

where the Kronecker delta $\delta\left(' j \neq x_{\kappa}\right.$ ') $\in\{1,0\}$ reflects whether or not the inequality $j \neq x_{\kappa}$ holds. On the other hand, setting

$$
\Xi:=\bigcup_{H \subseteq[m]}\{(H, \boldsymbol{\xi}) \mid \boldsymbol{\xi} \text { a refinement of a truncation of } G \text { by } H\}
$$

the right-hand side of (4.9) can be written as

$$
\sum_{H \subseteq[m]}(-1)^{m+\|H\|} c_{G, H} \alpha_{2 m+1}^{\uparrow H}\left(q^{-1}\right)=\sum_{(H, \xi) \in \Xi}(-1)^{m+\|H\|} \alpha_{2 m+1}^{\uparrow H}\left(q^{-1}\right)
$$

Now we explain why the last sum is indeed equal to the right-hand side of (4.10). Choosing an element $(H, \boldsymbol{\xi}) \in \Xi$, so that $\boldsymbol{\xi}=\left(\xi_{1}, \ldots, \xi_{k+1}\right)$ is a refinement of a truncation of $C(G)=\left(x_{1}, \ldots, x_{k+1}\right)$ by $C(H)=\left(y_{1}, \ldots, y_{\lambda}\right)$, is the same as fixing for each $\kappa \in[k+1]$ a truncation length $j_{\kappa} \in\left[x_{\kappa}\right]_{0}$ and a subset $K_{\kappa} \subseteq$ $\left[x_{\kappa}-j_{\kappa}-1\right]$, corresponding to a composition $\left(y_{\xi_{\kappa-1}+1}, \ldots, y_{\xi_{\kappa}}\right)$ of $x_{\kappa}-j_{\kappa}$. Moreover, the summands attached to the data $(H, \boldsymbol{\xi})$ in (4.11) and $\left(j_{\kappa}, K_{\kappa}\right)_{\kappa \in[k+1]}$ in (4.10) respectively agree:

$$
(-1)^{m+\|H\|}=(-1)^{\sum_{\kappa \in[k+1]} x_{\kappa}+\sum_{\kappa \in[k+1]}\left(\left|K_{\kappa}\right|+\delta\left({ }^{\prime} j \neq x_{\kappa}{ }^{\prime}\right)\right)}
$$


and

$$
\begin{aligned}
& \alpha_{2 m+1}^{\uparrow H}\left(q^{-1}\right)=\frac{\left(1-Y^{m}\right)\left(1-Y^{m-1}\right) \cdots(1-Y)}{\prod_{\iota \in[\lambda]}\left(1-Y^{y_{\iota}}\right)\left(1-Y^{y_{\iota}-1}\right) \cdots(1-Y)} \\
& =\frac{\left(1-Y^{m}\right)\left(1-Y^{m-1}\right) \cdots(1-Y)}{\prod_{\kappa \in[k+1]} \prod_{\iota=\xi_{\kappa-1}+1}^{\xi_{\kappa}}\left(1-Y^{y_{\iota}}\right)\left(1-Y^{y_{\iota}-1}\right) \cdots(1-Y)} \\
& =\frac{\left(1-Y^{m}\right)\left(1-Y^{m-1}\right) \cdots(1-Y)}{\prod_{\kappa \in[k+1]}\left(1-Y^{x_{\kappa}-j_{\kappa}}\right)\left(1-Y^{x_{\kappa}-j_{\kappa}-1}\right) \cdots(1-Y)} \prod_{\kappa \in[k+1]}\left(\begin{array}{c}
x_{\kappa}-j_{\kappa} \\
K_{\kappa}
\end{array}\right)_{Y} \\
& =\frac{\left(1-Y^{m}\right)\left(1-Y^{m-1}\right) \cdots(1-Y)}{\prod_{\kappa \in[k+1]}\left(1-Y^{x_{\kappa}}\right)\left(1-Y^{x_{\kappa}-1}\right) \cdots(1-Y)} \\
& \cdot \prod_{\kappa \in[k+1]}\left(\begin{array}{c}
x_{\kappa} \\
{\left[x_{\kappa-j_{\kappa}}, x_{\kappa}-1\right]}
\end{array}\right)_{Y}(1-Y)^{j_{\kappa}}\left(\begin{array}{c}
x_{\kappa}-j_{\kappa} \\
K_{\kappa}
\end{array}\right)_{Y} \\
& =\left(\begin{array}{l}
m \\
G
\end{array}\right)_{Y} \prod_{\kappa \in[k+1]}\left(\begin{array}{c}
x_{\kappa} \\
{\left[x_{\kappa-j_{\kappa}}, x_{\kappa}-1\right]}
\end{array}\right)_{Y}(1-Y)^{j_{\kappa}}\left(\begin{array}{c}
x_{\kappa}-j_{\kappa} \\
K_{\kappa}
\end{array}\right)_{Y} .
\end{aligned}
$$

This finishes the proof of (4.9) $)$. For later use we record

$$
\alpha_{2 m+1}^{\uparrow G}\left(q^{-1}\right) Y^{\sum_{\kappa \in[k+1]}\left(\begin{array}{c}
x_{\kappa} \\
2
\end{array}\right)}=\sum_{\substack{H \subseteq[m] \\
N(H)=m}}(-1)^{m+\|H\|} c_{G, H} \alpha_{2 m+1}^{\uparrow H}\left(q^{-1}\right) .
$$

Indeed, summing only over those $H \subseteq[m]$ such that $N(H)=m$ is achieved by setting persistently $j=j_{\kappa}=0$ in the above formulae. Clearly, under the restriction $j=0$ the term in the third line of (4.10) reduces to the left-hand side of (4.12).

Finally, we supply the proof of the formulae (i) above. We argue by induction on $i \in \mathbb{N}_{0}$. For $i=0$ we have

$$
Y^{\left(\begin{array}{l}
1 \\
2
\end{array}\right)}=1=\left(\begin{array}{l}
0 \\
\varnothing
\end{array}\right)_{Y}(Y-1)^{0} Y^{\left(\begin{array}{l}
0 \\
2
\end{array}\right),}
$$

and for $i>0$ we obtain, by induction,

$$
\begin{aligned}
& \sum_{j=0}^{i}\left(\begin{array}{c}
i \\
{[i-j, i-1]}
\end{array}\right)_{Y}(Y-1)^{j} Y^{\left(\frac{i-j}{2}\right)} \\
& =Y^{\left(\begin{array}{c}
i \\
2
\end{array}\right)}+\sum_{j \in[i]}\left(\begin{array}{c}
i \\
{[i-j, i-1]}
\end{array}\right)_{Y}(Y-1)^{j} Y^{\left(\begin{array}{c}
i-j \\
2
\end{array}\right)} \\
& =Y^{\left(\begin{array}{c}
i \\
2
\end{array}\right)}+\sum_{j \in[i]}\left(\begin{array}{c}
i-1 \\
{[i-j, i-2]}
\end{array}\right)_{Y}\left(\begin{array}{c}
i \\
i-1
\end{array}\right)_{Y}(Y-1)^{j} Y^{\left(\begin{array}{c}
i-j \\
2
\end{array}\right)} \\
& =Y^{\left(\begin{array}{c}
i \\
2
\end{array}\right)}+(Y-1)\left(\begin{array}{c}
i \\
i-1
\end{array}\right)_{Y} \sum_{j \in[i]}\left(\begin{array}{c}
i-1 \\
{[i-j, i-2]}
\end{array}\right)_{Y}(Y-1)^{j-1} Y^{\left(\begin{array}{c}
i-j \\
2
\end{array}\right)} \\
& =Y^{\left(\begin{array}{l}
i \\
2
\end{array}\right)}+(Y-1) \frac{\left(Y^{i}-1\right)}{(Y-1)} Y^{\left(\begin{array}{c}
i \\
2
\end{array}\right)}=Y^{i+\left(\begin{array}{c}
i \\
2
\end{array}\right)}=Y^{\left(\begin{array}{c}
i+1 \\
2
\end{array}\right)} \text {. }
\end{aligned}
$$


Proof of Proposition 4.3 (ii) for $n=2 m$ even. For $G \subseteq[m]$ we are looking to prove

$$
\alpha_{2 m, \varepsilon}^{\uparrow G}(q)=\varepsilon(-1)^{m} q^{m^{2}} \sum_{H \subseteq[m]}(-1)^{\|H\|} c_{G, H} \alpha_{2 m, \varepsilon}^{\uparrow H}\left(q^{-1}\right) .
$$

Again by an inductive argument, analogous to the case $n=2 m+1$, we may assume that in fact $N(G)=m$, i.e. $G \subseteq[m-1]$. Write $Y:=q^{-2}$ and $C(G)=\left(x_{1}, \ldots, x_{k+1}\right)$. Proposition 4.5 (ii) shows that in this case

$$
\alpha_{2 m, \varepsilon}^{\uparrow G}\left(q^{-1}\right)=\alpha_{2 m+1}^{\uparrow G}\left(q^{-1}\right)=\left(\begin{array}{c}
m \\
G
\end{array}\right)_{Y}
$$

in particular, as $\operatorname{deg}_{Y}\left(\begin{array}{c}m \\ G\end{array}\right)_{Y}=\left(\begin{array}{c}m \\ 2\end{array}\right)-\sum_{\kappa \in[k+1]}\left(\begin{array}{c}x_{\kappa} \\ 2\end{array}\right)$,

$$
\alpha_{2 m, \varepsilon}^{\uparrow G}(q)=\alpha_{2 m, \varepsilon}^{\uparrow G}\left(q^{-1}\right) Y^{-\left(\begin{array}{c}
m \\
2
\end{array}\right)+\sum_{\kappa \in[k+1]}\left(\begin{array}{c}
x_{\kappa} \\
2
\end{array}\right) .}
$$

We shall show below that

$$
\alpha_{2 m, \varepsilon}^{\uparrow G}\left(q^{-1}\right) Y^{\sum_{\kappa \in[k+1]}\left(\begin{array}{c}
x_{\kappa} \\
2
\end{array}\right)} q^{-m}=\varepsilon \sum_{H \subseteq[m]}(-1)^{m+\|H\|} c_{G, H} \alpha_{2 m, \varepsilon}^{\uparrow H}\left(q^{-1}\right) .
$$

From these equations, (4.13) follows readily.

It remains to prove (4.14). An easy computation gives

$$
\begin{aligned}
Y^{\sum_{\kappa \in[k+1]}\left(\begin{array}{c}
x_{\kappa} \\
2
\end{array}\right)} q^{-m} & =\varepsilon \frac{q^{-2 m}+\varepsilon q^{-m}}{1+\varepsilon q^{-m}} Y^{\sum_{\kappa \in[k+1]}\left(\begin{array}{c}
x_{\kappa} \\
2
\end{array}\right)} \\
& =\varepsilon \frac{1}{1+\varepsilon q^{-m}}\left(Y^{\sum_{\kappa \in[k+1]}\left(\begin{array}{c}
x_{\kappa}+1 \\
2
\end{array}\right)}+\varepsilon q^{-m} Y^{\sum_{\kappa \in[k+1]}\left(\begin{array}{c}
x_{\kappa} \\
2
\end{array}\right)}\right) \\
& =\varepsilon\left(\frac{Y^{\sum_{\kappa \in[k+1]}\left(\begin{array}{c}
x_{\kappa}+1 \\
2
\end{array}\right)}-Y^{\sum_{\kappa \in[k+1]}\left(\begin{array}{c}
x_{\kappa} \\
2
\end{array}\right)}}{1+\varepsilon q^{-m}}+Y^{\sum_{\kappa \in[k+1]}\left(\begin{array}{c}
x_{\kappa} \\
2
\end{array}\right)}\right) .
\end{aligned}
$$

From Proposition 4.5 we see that for $H \subseteq[m]$,

$$
\alpha_{2 m, \varepsilon}^{\uparrow H}\left(q^{-1}\right)= \begin{cases}\alpha_{2 m+1}^{\uparrow H}\left(q^{-1}\right) & \text { if } N(H)=m, \\ \alpha_{2 m+1}^{\uparrow H}\left(q^{-1}\right) /\left(1+\varepsilon q^{-m}\right) & \text { otherwise. }\end{cases}
$$

In view of (4.9) and (4.12), we thus obtain

$$
\begin{aligned}
\alpha_{2 m, \varepsilon}^{\uparrow G}\left(q^{-1}\right) Y^{\sum_{\kappa \in[k+1]}\left(\begin{array}{c}
x_{\kappa} \\
2
\end{array}\right)} q^{-m} & \\
= & \varepsilon \alpha_{2 m+1}^{\uparrow G}\left(q^{-1}\right)\left(\frac{Y^{\sum_{\kappa \in[k+1]}\left(\begin{array}{c}
x_{\kappa}+1 \\
2
\end{array}\right)}-Y^{\sum_{\kappa \in[k+1]}\left(\begin{array}{c}
x_{\kappa} \\
2
\end{array}\right)}}{1+\varepsilon q^{-m}}+Y^{\sum_{\kappa \in[k+1]}\left(\begin{array}{c}
x_{\kappa} \\
2
\end{array}\right)}\right) \\
= & \varepsilon \sum_{H \subseteq[m], N(H) \neq m}(-1)^{m+\|H\|} c_{G, H} \alpha_{2 m+1}^{\uparrow H}\left(q^{-1}\right) /\left(1+\varepsilon q^{-m}\right) \\
& +\varepsilon \sum_{H \subseteq[m], N(H)=m}(-1)^{m+\|H\|} c_{G, H} \alpha_{2 m+1}^{\uparrow H}\left(q^{-1}\right) \\
= & \varepsilon \sum_{H \subseteq[m]}(-1)^{m+\|H\|} c_{G, H} \alpha_{2 m, \varepsilon}^{\uparrow H}\left(q^{-1}\right) .
\end{aligned}
$$

This proves (4.14). 


\section{A CONJECTURE For the ORThogonal CASE}

In this section we discuss Conjecture $\mathrm{C}$ in more detail. As in Section 4, let $\mathcal{V}=(V, B, f)$ be an $n$-dimensional, non-degenerate quadratic space over the finite field $F=\mathbb{F}_{q}$. Our aim is to give, for $J \subseteq[n-1]$, an expression for the polynomial $\alpha_{\mathcal{V}}^{J}\left(q^{-1}\right)$ in terms of parabolic length functions on the Coxeter group $W$ of type $A_{n-1}$. If Conjecture $\mathrm{C}$ holds, the orthogonal case of Theorem $\mathrm{A}$ follows directly from Theorem 2.6.

Fix the Coxeter system $(W, S)$, where $W=\mathcal{S}_{n}$ and $S=\left\{s_{1}, \ldots, s_{n-1}\right\}$ denotes the standard set of Coxeter generators $s_{i}=(i \quad i+1), i \in[n-1]$. A crucial role is played by the following statistic on $W$.

Definition 5.1 (Length $L$ ). Recalling the notation from Section 2, for $w \in W$ set

$$
L(w):=\mathbf{b} \cdot \mathbf{l}_{\mathrm{R}}(w), \quad \text { where } \mathbf{b}=\left(b_{I}\right)_{I \subseteq S}=\left((-1)^{|I|} 2^{|S|-|I|-1}\right)_{I \subseteq S} .
$$

It is well known that the ordinary Coxeter length of a permutation $w \in W$ is equal to the number of inversion pairs associated to $w$, i.e. $l(w)=|\mathcal{I}(w)|$, where

$$
\mathcal{I}(w):=\left\{(i, j) \mid 1 \leq i<j \leq n, i^{w}>j^{w}\right\} .
$$

The parabolic length function $L$ also has a simple interpretation in terms of inversion pairs.

Lemma 5.2. For each $w \in W$,

$$
L(w)=|\{(i, j) \in \mathcal{I}(w) \mid i \not \equiv j \quad \bmod 2\}| .
$$

Proof. Let $w \in W$ and note that for any $I \subseteq[n-1]$,

$$
l_{\mathrm{R}}^{I}(w)=|\{(i, j) \in \mathcal{I}(w) \mid[i, j-1] \not I\}| .
$$

From this we derive

$$
\begin{aligned}
L(w) & =\frac{1}{2} \sum_{I \subseteq[n-1]}(-1)^{|I|} 2^{|S|-|I|} l_{\mathrm{R}}^{I}(w) \\
& =\frac{1}{2} \sum_{(i, j) \in \mathcal{I}(w)} \sum_{I \subseteq[n-1]}(-1)^{|I|} 2^{|S|-|I|} \delta\left(^{('}[i, j-1] \nsubseteq I^{\prime}\right) \\
& =\frac{1}{2} \sum_{(i, j) \in \mathcal{I}(w)}\left(\sum_{I \subseteq[n-1]}(-1)^{|I|} 2^{|S|-|I|}-\sum_{[i, j-1] \subseteq I}(-1)^{|I|} 2^{|S|-|I|}\right) \\
& =\frac{1}{2} \sum_{(i, j) \in \mathcal{I}(w)}\left((2-1)^{|S|}-(-1)^{j-i}(2-1)^{|S|-(j-i)}\right) \\
& =\frac{1}{2} \sum_{(i, j) \in \mathcal{I}(w)}\left(1-(-1)^{j-i}\right),
\end{aligned}
$$

where the Kronecker delta $\delta\left(^{\circ}[i, j-1] \nsubseteq I^{\prime}\right) \in\{1,0\}$ reflects whether or not the inclusion $[i, j-1] \nsubseteq I$ holds.

Definition 5.3 (Chessboard elements). We say that $w \in W$ is a chessboard element if $i+i^{w} \equiv j+j^{w}$ modulo 2 for all $i, j \in[n]$. Clearly, the set $\mathcal{C}_{n}$ of chessboard elements forms a subgroup of $W$. Note that $\mathcal{C}_{n}$ contains a subgroup $\mathcal{C}_{n, 0}$ consisting of elements $w$ such that $i \equiv i^{w}$ modulo 2 for all $i \in[n]$. If $n=2 m+1$ is odd, we 
have $\mathcal{C}_{n}=\mathcal{C}_{n, 0} \cong \mathcal{S}_{m+1} \times \mathcal{S}_{m}$. If $n=2 m$ is even, we have $\mathcal{C}_{n}=\left\langle w_{0}\right\rangle \ltimes \mathcal{C}_{n, 0}$, where $w_{0}$ denotes the longest element of $W$, and $\mathcal{C}_{n, 0} \cong \mathcal{S}_{m} \times \mathcal{S}_{m}$.

We write $\sigma: W \rightarrow\{1,-1\}, w \mapsto(-1)^{l(w)}$ for the sign character, and $\tau: \mathcal{C}_{n} \rightarrow$ $\{1,-1\}$ for the linear character with $\operatorname{ker}(\tau)=\mathcal{C}_{n, 0}$. Recall from the introduction that, in the even-dimensional case, we attach a sign $\varepsilon \in\{1,-1\}$ to $\mathcal{V}$. Observing that $\tau$ is trivial for $n$ odd, we define

$$
\chi_{\varepsilon}: \mathcal{C}_{n} \rightarrow\{1,-1\}, \chi_{\varepsilon}(w):= \begin{cases}\sigma(w) & \text { if } n \text { is odd, or if } n \text { is even and } \varepsilon=1, \\ \sigma(w) \tau(w) & \text { if } n \text { is odd, or if } n \text { is even and } \varepsilon=-1\end{cases}
$$

Conjecture C. For each $J \subseteq[n-1]$,

$$
\alpha_{\mathcal{V}}^{J}\left(q^{-1}\right)=\alpha_{n, \varepsilon}^{J}\left(q^{-1}\right)=\sum_{\substack{w \in \mathcal{C}_{n} \\ D_{\mathrm{L}}(w) \subseteq J}} \chi_{\varepsilon}(w) q^{-L(w)} .
$$

Note that, if Conjecture Cholds, the orthogonal case of Theorem $\AA$ follows from Theorem 2.6, equation (2.5), with $W^{\prime}=\mathcal{C}_{n}$, $\mathbf{b}$ as defined in (5.1) and $\chi=\chi_{\varepsilon}$. Conjecture $[$ has been confirmed for $|J| \leq 1$ and verified for $n \leq 13$.

Example 5.4. For $n=3$, the explicit data is given in Table 4 .

TABLE 4

\begin{tabular}{|l|c|c|c|c|}
\hline$J \subseteq[2]$ & $\varnothing$ & $\{1\}$ & $\{2\}$ & $\{1,2\}$ \\
\hline \hline$a_{3}^{J}(q)$ & 1 & $q^{2}$ & $q^{2}$ & $q^{3}-q$ \\
\hline$\alpha_{3}^{J}\left(q^{-1}\right)$ & 1 & 1 & 1 & $1-q^{-2}$ \\
\hline
\end{tabular}

For $w \in W=\left\langle s_{1}, s_{2}\right\rangle=\mathcal{S}_{3}$, the statistic $L(w)=2 l(w)-l_{\mathrm{R}}^{\{1\}}(w)-l_{\mathrm{R}}^{\{2\}}(w)$, the character $\chi_{\varepsilon}(w)=\sigma(w)=(-1)^{l(w)}$ and the left descent set $D_{\mathrm{L}}(w)$ take the values in Table 5.

\section{TABLE 5}

\begin{tabular}{|l|c|c|c|c|c|c|}
\hline$w$ & id & $s_{1}$ & $s_{2}$ & $s_{1} s_{2}$ & $s_{2} s_{1}$ & $s_{1} s_{2} s_{1}$ \\
\hline \hline$L(w)$ & 0 & 1 & 1 & 1 & 1 & 2 \\
\hline$\chi_{\varepsilon}(w)$ & 1 & -1 & -1 & 1 & 1 & -1 \\
\hline$D_{\mathrm{L}}(w)$ & $\varnothing$ & $\{1\}$ & $\{2\}$ & $\{1\}$ & $\{2\}$ & $\{1,2\}$ \\
\hline
\end{tabular}

If $n$ is odd or if $n$ is even and $\varepsilon=1$, the character $\chi_{\varepsilon}$ naturally extends to the sign character on the whole group $W$. Interestingly, in this case also a modified version of equation (5.2) seems to hold, where $\chi_{\varepsilon}$ is replaced by $\sigma$ and one sums over all elements $w \in W$. In fact, we originally introduced chessboard elements in an attempt to control cancellation in this larger sum. Evidently, the contributions of any two elements $w_{1}, w_{2} \in W$ with $w_{1}^{-1} w_{2} \in S$ and $L\left(w_{1}\right)=L\left(w_{2}\right)$ cancel each other. Therefore we were led to sum over the set

$$
\mathcal{M}:=\left\{w \in W \mid \forall s \in S: D_{\mathrm{L}}(w) \neq D_{\mathrm{L}}(w s) \text { or } L(w) \neq L(w s)\right\} .
$$

The set $\mathcal{M}$ is easily seen to be closed under right-multiplication by the longest element $w_{0}$ and might indeed coincide with $\mathcal{C}_{n}$. Aided by computer evidence, we distilled Conjecture $\mathrm{C}$ out of this circle of ideas. 


\section{APPENDiX A. EXPLicit EXAMPLES}

Here we collect a few examples of Igusa-type functions for the family

$$
\mathbf{F}=\left(F_{J}(\mathbf{X})\right)_{J \subseteq[n-1]}=\left(\prod_{i \in J} \frac{X_{i}}{1-X_{i}}\right)_{J \subseteq[n-1]} .
$$

A.1. A flag of alternating bilinear forms. Let $V$ be a vector space of dimension $n=6$, equipped with a flag of alternating bilinear forms $\boldsymbol{B}$ of type $I=\{4\}$. This kind of example was considered immediately after Definition 3.2. The explicit data is given in Table 6 .

TABLE 6

\begin{tabular}{|l|c|c|c|c|}
\hline$J \subseteq[4]$ & $\varnothing$ & $\{2\}$ & $\{4\}$ & $\{2,4\}$ \\
\hline \hline$a_{6,\{4\}}^{J}(q)$ & 1 & $q^{8}+q^{4}+q^{2}$ & $q^{8}+q^{6}+1$ & $q^{2}\left(q^{12}-1\right) /\left(q^{2}-1\right)$ \\
\hline$\alpha_{6,\{4\}}^{J}\left(q^{-1}\right)$ & 1 & $1+q^{-4}+q^{-6}$ & $1+q^{-2}+q^{-8}$ & $\left(1-q^{-12}\right) /\left(1-q^{-2}\right)$ \\
\hline
\end{tabular}

Thus, in view of Definition 3.4

$$
\operatorname{Ig}_{(V, \boldsymbol{B})}\left(q^{-1}, \mathbf{X}\right)=\frac{1+\left(q^{-4}+q^{-6}\right) X_{1}+\left(q^{-2}+q^{-8}\right) X_{2}+q^{-10} X_{1} X_{2}}{\left(1-X_{1}\right)\left(1-X_{2}\right)} .
$$

Note that, in accordance with Theorem B.

$$
\operatorname{Ig}_{(V, \boldsymbol{B})}\left(q, X_{1}^{-1}, X_{2}^{-1}\right)=q^{10} \operatorname{Ig}_{(V, \boldsymbol{B})}\left(q^{-1}, X_{2}, X_{1}\right) .
$$

\section{A.2. Quadratic spaces.}

A.2.1. Odd dimension. Let $V$ be a vector space of odd dimension $n=3$, equipped with a non-degenerate quadratic form $f$. The explicit data for this case is given in Table 7 .

\section{TABLE 7}

\begin{tabular}{|l|c|c|c|}
\hline$J \subseteq[2]$ & $\varnothing$ & $\{1\},\{2\}$ & $\{1,2\}$ \\
\hline \hline$a_{3}^{J}(q)$ & 1 & $q^{2}$ & $q^{3}-q$ \\
\hline$\alpha_{3}^{J}\left(q^{-1}\right)$ & 1 & 1 & $1-q^{-2}$ \\
\hline
\end{tabular}

In view of Definition 4.1 ,

$$
\operatorname{Ig}_{3}\left(q^{-1}, \mathbf{X}\right)=\frac{1-q^{-2} X_{1} X_{2}}{\left(1-X_{1}\right)\left(1-X_{2}\right)}
$$

and this satisfies the functional equation predicted by Theorem $\mathrm{A}$. 
TABLE 8

\begin{tabular}{|l|c|c|c|c|c|}
\hline$J \subseteq[3]$ & $\varnothing$ & $\{1\},\{3\}$ & $\{2\}$ & $\{1,2\},\{2,3\},\{1,3\}$ & $\{1,2,3\}$ \\
\hline \hline$a_{4,1}^{J}(q)$ & 1 & $q^{3}-q$ & $q^{4}+q^{2}$ & $q^{5}-q^{3}$ & $q^{2}\left(q^{2}-1\right)^{2}$ \\
\hline$\alpha_{4,1}^{J}\left(q^{-1}\right)$ & 1 & $1-q^{-2}$ & $1+q^{-2}$ & $1-q^{-2}$ & $\left(1-q^{-2}\right)^{2}$ \\
\hline$a_{4,-1}^{J}(q)$ & 1 & $q^{3}+q$ & $q^{4}+q^{2}$ & $q^{5}+q^{3}$ & $q^{2}\left(q^{4}-1\right)$ \\
\hline$\alpha_{4,-1}^{J}\left(q^{-1}\right)$ & 1 & $1+q^{-2}$ & $1+q^{-2}$ & $1+q^{-2}$ & $1-q^{-4}$ \\
\hline
\end{tabular}

A.2.2. Even dimension. Let $V$ be a vector space of dimension $n=4$, equipped with a non-degenerate quadratic form $f$. The explicit data for this case is given in Table 8.

Note that, for both values of $\varepsilon$, the number of distinct polynomials among the $\alpha_{4, \varepsilon}^{J}\left(q^{-1}\right)$ is comparatively small. This illustrates that the map $J \mapsto \alpha_{n, \varepsilon}^{J}\left(q^{-1}\right)$ factors over the bisecting map $\varphi$; cf. Proposition 4.5. One readily computes

$$
\begin{aligned}
\operatorname{Ig}_{4,1}\left(q^{-1}, \mathbf{X}\right) & =\frac{1+q^{-2}\left(-X_{1} X_{2}+X_{1} X_{3}-X_{2} X_{3}-X_{1}+X_{2}-X_{3}\right)+q^{-4} X_{1} X_{2} X_{3}}{\left(1-X_{1}\right)\left(1-X_{2}\right)\left(1-X_{3}\right)}, \\
\operatorname{Ig}_{4,-1}\left(q^{-1}, \mathbf{X}\right) & =\frac{1+q^{-2}\left(-X_{1} X_{2}-X_{1} X_{3}-X_{2} X_{3}+X_{1}+X_{2}+X_{3}\right)-q^{-4} X_{1} X_{2} X_{3}}{\left(1-X_{1}\right)\left(1-X_{2}\right)\left(1-X_{3}\right)} .
\end{aligned}
$$

These Igusa-type functions satisfy the functional equations predicted by Theorem $\mathrm{A}$.

A.3. A symmetric bilinear space in characteristic 2. Let $V=\left\langle e_{1}, e_{2}, e_{3}, e_{4}\right\rangle$ be a 4 -dimensional vector space over a finite field $F=\mathbb{F}_{q}$ with $\operatorname{char} F=2$. Let $B$ denote the non-degenerate symmetric bilinear form on $V$ such that $B\left(e_{i}, e_{j}\right)=\delta_{i j}$, and define $f: V \rightarrow F$ by $f(x):=B(x, x)$. We consider flags of non-degenerate symmetric bilinear subspaces in the non-degenerate symmetric bilinear space $\mathcal{V}:=$ $(V, B, f)$. For $J \subseteq\{1,2,3\}$ let $a_{\mathcal{V}}^{J}(q)$ denote the number of non-degenerate flags of type $J$ in $\mathcal{V}$, and define the normalised polynomials $\alpha_{\mathcal{V}}^{J}\left(q^{-1}\right)$ and the 'Igusatype function' $\operatorname{Ig}_{\mathcal{V}}\left(q^{-1}, \mathbf{X}\right)$ accordingly. The explicit data for this case is given in Table 9 .

TABLE 9

\begin{tabular}{|l|c|c|c|c|c|}
\hline$J \subseteq[3]$ & $\varnothing$ & $\{1\},\{3\}$ & $\{2\}$ & $\{1,2\},\{2,3\},\{1,3\}$ & $\{1,2,3\}$ \\
\hline \hline$a_{\mathcal{V}}^{J}(q)$ & 1 & $q^{3}$ & $q^{4}+q^{2}$ & $q^{5}$ & $q^{4}\left(q^{2}-1\right)$ \\
\hline$\alpha_{\mathcal{V}}^{J}\left(q^{-1}\right)$ & 1 & 1 & $1+q^{-2}$ & 1 & $1-q^{-2}$ \\
\hline
\end{tabular}

The associated 'Igusa-type function'

$$
\operatorname{Ig}_{\mathcal{V}}\left(q^{-1}, \mathbf{X}\right)=\frac{1+q^{-2} X_{2}\left(1-X_{1}-X_{3}\right)}{\left(1-X_{1}\right)\left(1-X_{2}\right)\left(1-X_{3}\right)}
$$

does not satisfy a functional equation; this illustrates our remarks at the end of the Introduction.

\section{REFERENCES}

1. E. Artin, Geometric algebra, Interscience Publishers, New York, 1957. MR0082463 (18:553e)

2. P. Cameron, Projective and polar spaces, QMW Maths Notes 13, Queen Mary and Westfield College, London, 1991. MR1153019 (93c:51011) 
3. J. Denef and D. Meuser, A functional equation for Igusa's local zeta function, Amer. J. Math. 113 (1991), no. 6, 1135-1152. MR 1137535 (93e:11145)

4. M.P.F. du Sautoy, Zeta functions of groups: The quest for order versus the flight from ennui, Groups St. Andrews 2001 in Oxford, Cambridge Univ. Press, Cambridge, 2003. MR2051525 (2005i:20035)

5. M.P.F. du Sautoy and F. Grunewald, Analytic properties of zeta functions and subgroup growth, Ann. of Math. (2) 152 (2000), 793-833. MR1815702 (2002h:11084)

6. M.P.F. du Sautoy and F. Grunewald, Zeta functions of groups and rings, International Congress of Mathematicians. Vol. II, 131-149, Eur. Math. Soc., Zürich, 2006. MR2275592 (2008d:11102)

7. M.P.F. du Sautoy and A. Lubotzky, Functional equations and uniformity for local zeta functions of nilpotent groups, Amer. J. Math. 118 (1994), no. 1, 39-90. MR.1375303 (97g:11137)

8. R.L. Graham, D.E. Knuth, and O. Patashnik, Concrete mathematics, Addison Wesley, Reading, MA, 1998. MR:1678922 (99m:68002)

9. L.C. Grove, Classical groups and geometric algebra, Graduate Studies in Mathematics 39, AMS, Providence, RI, 2002. MR1859189 (2002m:20071)

10. J.E. Humphreys, Reflection groups and Coxeter groups, Cambridge Studies in Advanced Mathematics 29, Cambridge Univ. Press, Cambridge, 1990. MR.1066460 (92h:20002)

11. J.-I. Igusa, Universal p-adic zeta functions and their functional equations, Amer. J. Math. 111 (1989), 671-716. MR1020825 (91e:11142)

12. B. Klopsch and C. Voll, Counting subgroups of the higher Heisenberg groups, unpublished.

13. J. Milnor and D. Husemoller, Symmetric bilinear forms, Ergebnisse der Mathematik und ihrer Grenzgebiete, vol. 73, Springer-Verlag, Berlin, 1973. MR0506372 (58:22129)

14. A. Pfister, Quadratic forms with applications in algebraic geometry and topology, London Mathematical Society Lecture Note Series, vol. 217, Cambridge Univ. Press, Cambridge, 1995. MR:1366652 (97c:11046)

15. R. Scharlau, Buildings, Handbook of incidence geometry (F. Buekenhout, ed.), North-Holland, Amsterdam, 1995. MR 1360715 (96e:51002)

16. R.P. Stanley, Enumerative combinatorics, Cambridge Studies in Advanced Mathematics 49, vol. I, Cambridge Univ. Press, Cambridge, 1997. MR.1442260 (98a:05001)

17. R.P. Stanley, Hilbert functions of graded algebras, Advances in Math. 28 (1987), no. 1, 57-83. MR 0485835 (58:5637)

18. C. Voll, Functional equations for local normal zeta functions of nilpotent groups, Geom. Funct. Anal. (GAFA) 15 (2005), 274-295, with an appendix by A. Beauville. MR2140633 (2006k:20065)

19. - Counting subgroups in a family of semi-direct nilpotent products, Bull. London Math. Soc. 38 (2006), no. 5, 743-752. MR.2268358 (2007i:11123)

20. _ Functional equations for zeta functions of groups and rings, Ann. of Math., to appear.

Mathematisches Institut, Heinrich-Heine-Universität, D-40225 Düsseldorf, Germany Current address: Department of Mathematics, Royal Holloway, University of London, Egham TW20 0EX, United Kingdom

E-mail address: Benjamin.Klopsch@rhul.ac.uk

Max-Planck-Institut für Mathematik, Vivatsgasse 7, D-53111 Bonn, Germany Current address: School of Mathematics, University of Southampton, Southampton SO17 1BJ, United Kingdom

E-mail address: C.Voll.98@cantab.net 